ARTICLE

\title{
Oncogenic cooperation between TCF7-SPI1 and NRAS(G12D) requires $\beta$-catenin activity to drive T- cell acute lymphoblastic leukemia
}

Quentin Van Thillo (10) 1,2,3,17, Jolien De Bie 1,2,4,17, Janith A. Seneviratne (i) ${ }^{5,6}$, Sofie Demeyer ${ }^{1,2,3}$, Sofia Omari (5,6, Anushree Balachandran ${ }^{5,6}$, Vicki Zhai ${ }^{5,6}$, Wai L. Tam7, Bram Sweron ${ }^{1,2}$, Ellen Geerdens ${ }^{1,2,3}$, Olga Gielen ${ }^{1,2}$, Sarah Provost ${ }^{1,2}$, Heidi Segers ${ }^{3,8,9}$, Nancy Boeckx ${ }^{8,10}$, Glenn M. Marshall ${ }^{5,6,11}$, Belamy B. Cheung ${ }^{5,6}$, Kiyotaka Isobe ${ }^{12}$, Itaru Kato (D) ${ }^{12}$, Junko Takita ${ }^{12}$, Timothy G. Amos (D) ${ }^{13}$, Ira W. Deveson (1) ${ }^{13,14}$, Hannah McCalmont ${ }^{5,6}$, Richard B. Lock ${ }^{5,6}$, Ethan P. Oxley ${ }^{15}$, Maximilian M. Garwood ${ }^{15}$, Ross A. Dickins (iD) ${ }^{15}$, Anne Uyttebroeck (10) ${ }^{3,8,9}$, Daniel R. Carter $5,6,16$, Jan Cools (i) $1,2,3 凶 \&$ Charles E. de Bock (iD ${ }^{5,6}$ 布

Spi-1 Proto-Oncogene (SPI1) fusion genes are recurrently found in T-cell acute lymphoblastic leukemia (T-ALL) cases but are insufficient to drive leukemogenesis. Here we show that SPI1 fusions in combination with activating NRAS mutations drive an immature T-ALL in vivo using a conditional bone marrow transplant mouse model. Addition of the oncogenic fusion to the NRAS mutation also results in a higher leukemic stem cell frequency. Mechanistically, genetic deletion of the $\beta$-catenin binding domain within Transcription factor 7 (TCF7)-SPI1 or use of a $\mathrm{TCF} / \beta$-catenin interaction antagonist abolishes the oncogenic activity of the fusion. Targeting the TCF7-SPI1 fusion in vivo with a doxycycline-inducible knockdown results in increased differentiation. Moreover, both pharmacological and genetic inhibition lead to downregulation of SPI1 targets. Together, our results reveal an example where TCF7-SPI1 leukemia is vulnerable to pharmacological targeting of the TCF/ $\beta$-catenin interaction.

\footnotetext{
${ }^{1}$ Department of Human Genetics, KU Leuven, Leuven, Belgium. ${ }^{2}$ Center for Cancer Biology, VIB, Leuven, Belgium. ${ }^{3}$ Leuvens Kanker Instituut (LKI), KU Leuven - UZ Leuven, Leuven, Belgium. ${ }^{4}$ Center for Human Genetics, UZ Leuven, Leuven, Belgium. ${ }^{5}$ Children's Cancer Institute, UNSW Sydney, Lowy Cancer Research Centre, Sydney, NSW, Australia. ${ }^{6}$ School of Women's and Children's Health, UNSW Sydney, Sydney, NSW, Australia. ${ }^{7}$ Technology Innovation Lab, VIB, Gent, Belgium. ${ }^{8}$ Department of Oncology, KU Leuven, Leuven, Belgium. ${ }^{9}$ Department of Pediatric Hemato-Oncology, UZ Leuven, Leuven, Belgium.

${ }^{10}$ Department of Laboratory Medicine, UZ Leuven, Leuven, Belgium. ${ }^{11}$ Kids Cancer Centre, Sydney Children's Hospital, Randwick, NSW, Australia.

${ }^{12}$ Department of Pediatrics, Graduate School of Medicine, Kyoto University, Kyoto, Japan. ${ }^{13}$ Kinghorn Centre for Clinical Genomics, Garvan Institute of Medical Research, Sydney, NSW, Australia. ${ }^{14}$ St Vincent's Clinical School, Faculty of Medicine, UNSW Sydney, Sydney, NSW, Australia. ${ }^{15}$ Australian Centre for Blood Diseases, Monash University, Melbourne, VIC, Australia. ${ }^{16}$ School of Biomedical Engineering, University of Technology, Sydney, NSW, Australia.

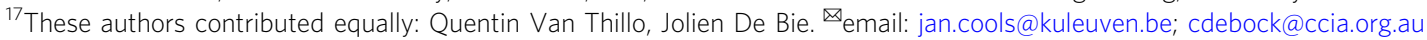


T -cell acute lymphoblastic leukemia or T-ALL is a hematopoietic malignancy of T-cell precursor cells that primarily affects young children. Chromosomal rearrangements are present in the majority of T-ALL patients, resulting in the ectopic expression of transcription factors, including T-cell acute lymphocytic leukemia protein 1 (TAL1), Tcell leukemia homeobox protein 1 and 3 (TLX1 and TLX3) and NK2 homeobox 1 (NKX2-1). Other typical pathways involved in the pathogenesis of T-ALL include the NOTCH1, Janus kinase (JAK)/signal transducer of activation and transcription (STAT) and RAS signaling pathways ${ }^{1-5}$. Often multiple cooperating hits are needed to induce leukemia ${ }^{6-8}$.

Seki et al. recently reported that up to $4 \%$ of pediatric T-ALL patients carry gene fusions involving the SPI1 locus on chromosome 11 (also known as PU.1) with different fusion partners, and that patients harboring such SPII fusions represent a distinct subgroup of T-ALL cases with significantly shorter overall survival ${ }^{9}$. While SPI1 (PU.1) is an important transcription factor in all hematopoietic cells, TCF7 (located on the short arm of chromosome 5) encodes TCF1, a T-cell specific transcriptional activator that is highly expressed in the earliest thymic progenitors and controls chromatin accessibility of $\mathrm{T}$-cell regulatory elements ${ }^{10,11}$. Dysregulation of PU.1 contributes to several types of leukemia ${ }^{12-18}$ and forced overexpression of SPI1 in T-cell progenitors is known to inhibit T-cell differentiation ${ }^{19-21}$. Conversely, TCF7 knock-out also leads to a T-cell differentiation block $^{22}$.

In normal T-cell development, NOTCH-signaling plays an important role in downregulating PU.1 and upregulating TCF1 expression during progression from the double negative (DN) 2 to DN3 T-cell stage $20,23,24$. To evaluate the role of SPI1 fusion genes in T-ALL, Seki et al. expressed distinct fusion transcripts in mouse stem/progenitor cells and documented an increased T-cell proliferation and a maturation block at the DN3 stage upon SPI1 or SPI1 fusion expression. Constitutive expression of TCF7-SPI1 alone, however, was not found to cause leukemia development in a mouse bone marrow transplant model ${ }^{9}$. Significantly, whilst TCF7-SPI1 fusions are insufficient for frank T-cell leukemia development, these clinical cases are often coincident with NRAS mutations suggesting the potential for oncogenic cooperation. Here we show that TCF7-SPI1 fusions and the NRAS(G12D) activating mutation cooperate to form an immature T-ALL in vivo that is absent when each is expressed alone. Moreover, using a genetic approach we show this cooperation absolutely requires the presence of the $\beta$-catenin interaction domain in the TCF7-SPI1 fusion with genetic loss of the fusion resulting in immunophenotypic differentiation. We also provide proof-ofprinciple that this is phenocopied through small molecule antagonism of the interaction between $\beta$-catenin and TCF1 of the TCF7-SPI1 fusion.

\section{Results}

Identification of a TCF7-SPI1 positive and NRAS mutant TALL case. Peripheral blood cells from patient X09, a 6-year old boy newly diagnosed with an immature CD4/CD8 double negative TALL, underwent whole-genome and RNA sequencing, revealing a translocation between chromosomes 5 and 11 and co-occurring mutations in NRAS, NOTCH1, Krüppel-like factor 9 (KLF9), Cyclindependent kinase inhibitor $2 A$ (CDKN2A), Sidekick cell adhesion molecule 1 (SDK1), T-cell receptor gamma (TRG), and transducin (beta)-like 1 X-linked receptor 1 (TBL1XR1) (Fig. 1a). The $\mathrm{t}(5 ; 11)$ translocation breakpoint occurred at TCF7 intron 4-5 (Chr. 5) and SPI1 intron 2-3 (Chr. 11) generating a predicted fusion transcript connecting exons 1-4 from TCF7 to exons 3-5 of SPI1 that was validated using both long-read nanopore and Sanger sequencing (Fig. 1b, c). At the protein level, the predicted fusion product brings together the first 182 amino acids from TCF1 (TCF7), including the $\mathrm{N}$-terminal $\beta$-catenin binding region, and the terminal amino acids 52 to 271 of PU.1 (SPI1), resulting in a 402 amino acid long protein that still retains the DNA binding domain regions of PU.1 (Fig. 1d).

Single-cell RNA sequencing identifies that high SPI1 expression is associated with NRAS, stem cell, and Wnt/ $\beta$-catenin signaling cell signatures. Single-cell RNA sequencing was carried out within six primary T-ALL samples (Supplementary Data 1-4) including patient X09 to identify transcriptomic determinants of this T-ALL subtype. Clustering a total of 13,848 cells from the pooled samples identified 22 cell clusters representing either malignant T cells or normal cells including NK-T cells, B cells, monocytes, and red blood cells (Supplementary Fig. 1a-c). Focusing on the 11,620 malignant $\mathrm{T}$ cells only, patients with TCF7-SPI1 fusions were distinct from the other T-ALL samples and displayed significantly higher SPI1 expression (Fig. 2a-c, Supplementary Fig. 1d). Since SPI1 is the $3^{\prime}$ partner of the TCF7SPI1 fusion, and $10 \times$ short read RNA-sequencing is $3^{\prime}$ biased, we confirmed that SPI1 expression levels were correlated with TCF7SPI1 fusion expression through long read nanopore sequencing (Supplementary Fig. 1e-h, Supplementary Data 5). Gene expression analysis also showed that SPI1 expression in fusion positive cases correlated with higher levels of hematopoietic stem markers like CD34 and lower levels of mature T-cell markers like $C D 3 D$ and $C D 1 E$ (Fig. $2 \mathrm{~d}-\mathrm{g}$ ) reconciling with the immature phenotype by flow cytometry analysis.

We next sought to determine how fusion positive cases compared to other defined T-ALL subtypes at the transcriptome level ${ }^{5}$. We found fusion cases clustered in close proximity to the early T- cell precursor (ETP) and immature Lim domain only $2 /$ Lymphoblastic Leukemia Associated Hematopoiesis Regulator 1 (LMO2/LYL1) cases of the Liu et al. dataset (Supplementary Fig. 2a, b), suggesting fusion cases have a transcriptome similar to ETP-ALL despite their immunophenotype. Therefore, using published transcriptome data ${ }^{5,25}$, we next generated gene signatures from ETP- and nonETP-ALL patients (Supplementary Data 6,7$)$. The resulting Liu and Zhang ETP-ALL signatures were validated using single-cell RNA-sequencing data of normal developing human thymocytes ${ }^{26}$, confirming highest enrichment in cells within the designated ETP region. Conversely, the Liu nonETP-ALL and Zhang T-ALL signatures had the highest enrichment in $\mathrm{T}$ cells designated at double positive (DP) and $\alpha \beta T$-entry regions (Supplementary Fig. 2c-k). Applying these signatures to our data, the three TCF7-SPI1 fusion positive cases were positively enriched for an ETP-ALL signature and negatively enriched for the complementary T-ALL signature (Fig. 2h, i, Supplementary Fig. 3a-f).

Combining additional bulk RNA-sequencing data of these 6 patients with the cohort described by Seki et al. further revealed that patients harboring both the TCF7-SPI1 fusion and NRAS mutations had a higher ETP-ALL signature compared to those with the TCF7-SPI1 fusion alone (Supplementary Fig. 4). We also used GSEA analysis to determine signaling pathways associated with SPI1 expression, which revealed a positive enrichment for myeloid, stem, and $\mathrm{Wnt} / \beta$-catenin gene signatures (Fig. 2j, Supplementary Data 8). Collectively, these data highlight the fusion positive transcriptomes are enriched for an ETP signature and there is a strong link between TCF7-SPI1 fusion status, RAS activation and the $\mathrm{Wnt} / \beta$-catenin pathway.

TCF7-SPI1 requires mutant NRAS to cause in vitro transformation. A recent study identified recurrent translocations similar to our TCF7-SPI1 fusion and these often harbored mutations in either NRAS $\left(3 / 7\right.$ cases) or KRAS $(1 / 7 \text { cases })^{9}$. This same study 
a

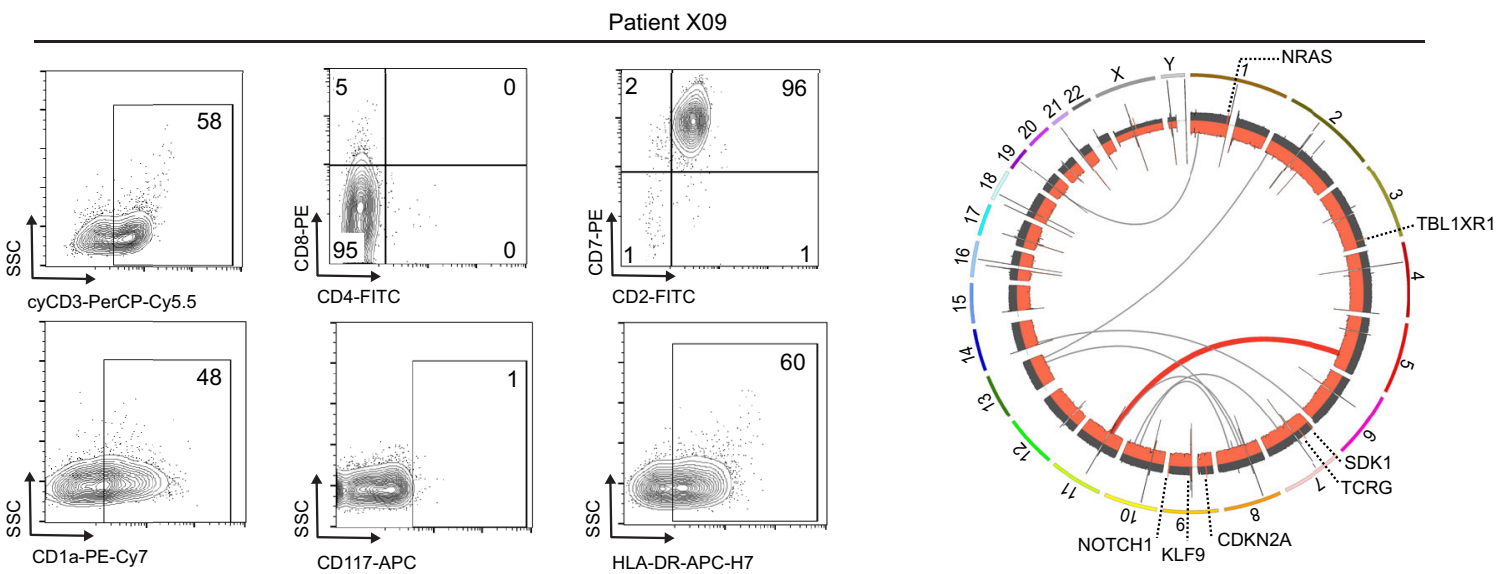

b

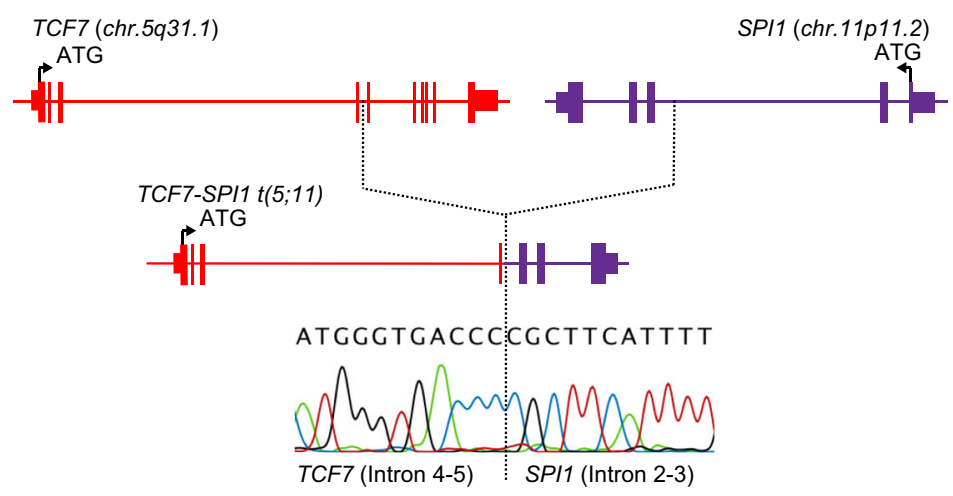

d
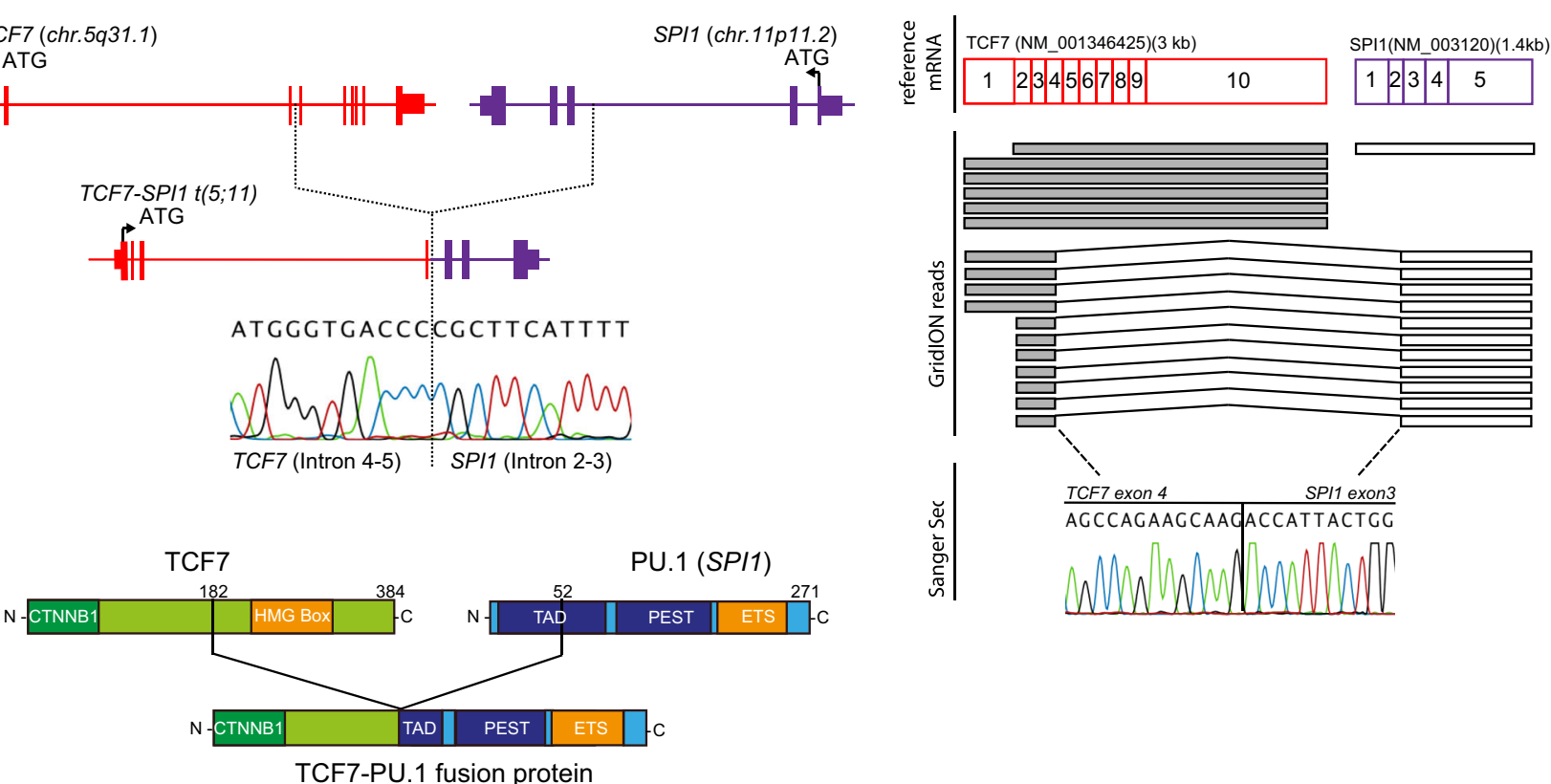

Fig. 1 Identification and characterization of the TCF7-SPI1 fusion in patient X09 with T-cell acute lymphoblastic leukemia. a Left panel: flow cytometry staining of the X09 patient sample for cyCD3, CD1a, CD4, CD8, CD117, CD2, CD7, HLA-DR. Right panel: Circos plot showing the translocation between chromosomes 5 and 11 in the X09 patient. Additional mutations are shown with their respective chromosomal location. $\mathbf{b}$ Scheme of the t(5;11) translocation between TCF7 and SPI1. The breakpoints are located at TCF7 intron 4-5 (Chr. 5) and SPI1 intron 2-3 (Chr. 11). c Long read nanopore sequencing confirming the translocation with the resulting GridION reads shown schematically alongside Sanger sequencing confirmation of the breakpoint region. $\mathbf{d}$ Schematic representation of the resulting TCF1-PU.1 (TCF7-SPI1) fusion protein. The different domains are shown. CTNNB1: $\beta$-catenin binding domain; TAD: trans-activating domain; HMG: high mobility group box.

showed that expression of SPI1 fusions using an in vivo bone marrow transplant model led to a block in T-cell differentiation but not acute leukemia ${ }^{9}$. Given the high frequency of cooccurring mutations with NRAS (OR 5.833, p value 0.0067, Supplementary Data 9) and association with NRAS signaling (Fig. 2j) we sought to investigate whether the TCF7-SPI1 fusion cooperates with oncogenic NRAS to drive T-ALL. We generated two sets of retroviral vectors that would express TCF7-SPI1 and/ or NRAS(G12D) from a single vector using a viral P2A sequence, in either a constitutive or inducible manner as has been previously described ${ }^{6}$ (Fig. 3a, b). Western blot analysis confirmed efficient constitutive or inducible expression of both the TCF7SPI1 (TCF1-PU.1) fusion and/or NRAS(G12D) upon transduction of $\mathrm{Ba} / \mathrm{F} 3$ cells and $\mathrm{Ba} / \mathrm{F} 3$-Cre cells respectively (Fig. $3 \mathrm{c}, \mathrm{d}$ ). The transformation potential was first determined in wild-type and Cre-positive Ba/F3 cells expressing NRAS(G12D) alone or in combination with TCF7-SPI1. Only when NRAS(G12D) was present, were the cells able to grow in an interleukin-3 (Il3)independent manner, demonstrating that the NRAS(G12D) protein provides the essential proliferation and survival signals to lymphoid cells (Fig. 3e, f). Using a more physiological relevant Cre-positive pro- $T$ cell culture model in combination with our inducible vector system, all transduced cells grew in the presence of Delta-like ligand 4 (Dll4), interleukin-7 (Il7) and stem cell factor (Scf). Notably, inducible expression of NRAS (G12D) + /- TCF7-SPI1 provided growth and survival signals that could transform cells to Scf- and to a lesser extent Il7independent growth, but all remained dependent on Notch signaling via Dll4 (Fig. $3 g-j)$. Consequently, these results show that $N R A S(G 12 D)$ alone is sufficient and necessary for transformation of these two in vitro cellular systems to cytokine-independent growth. 

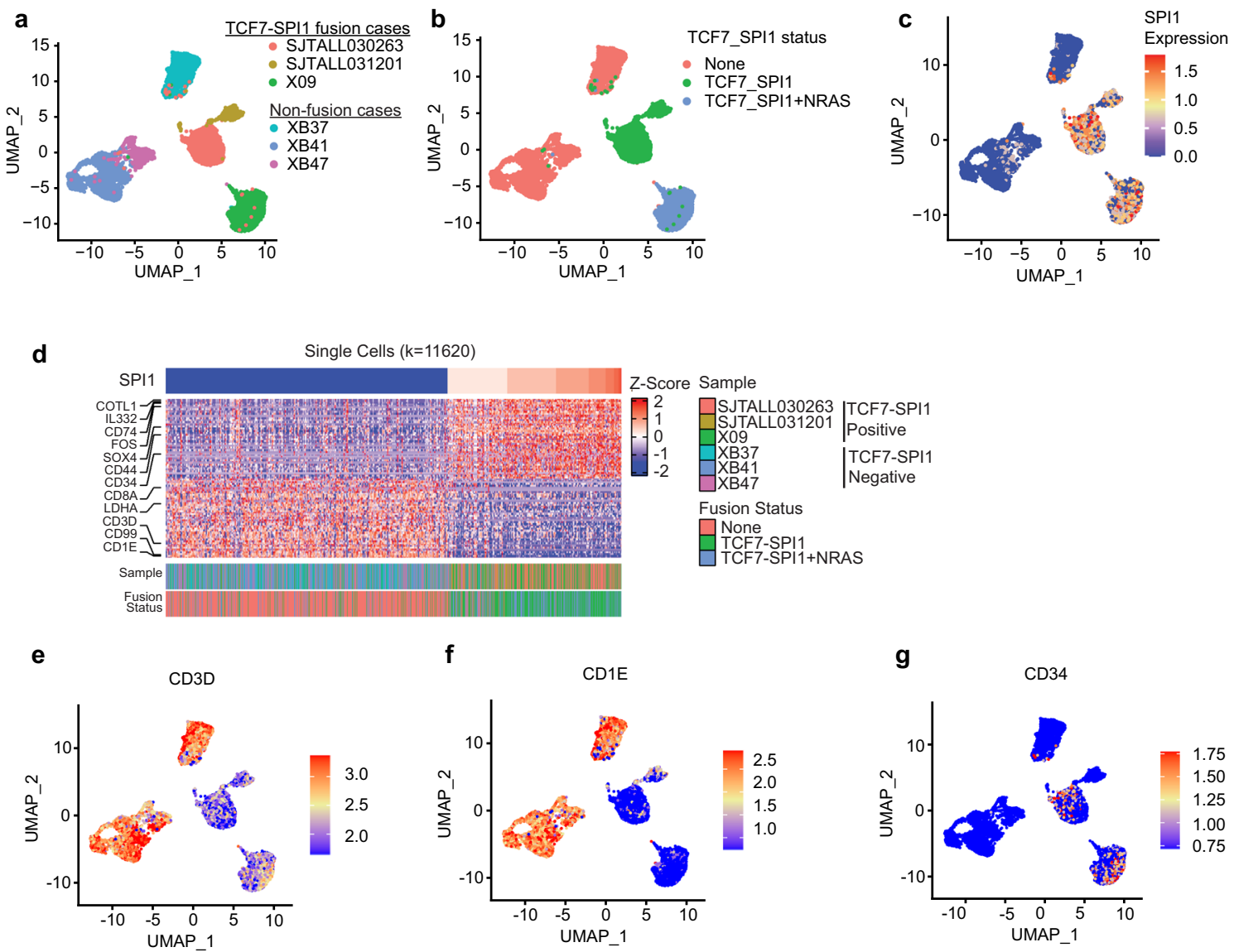

f
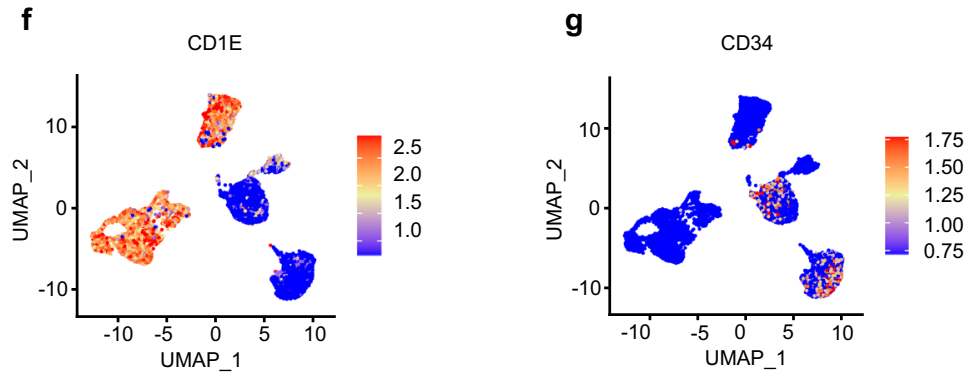

h

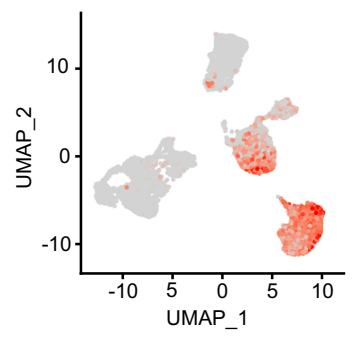

i
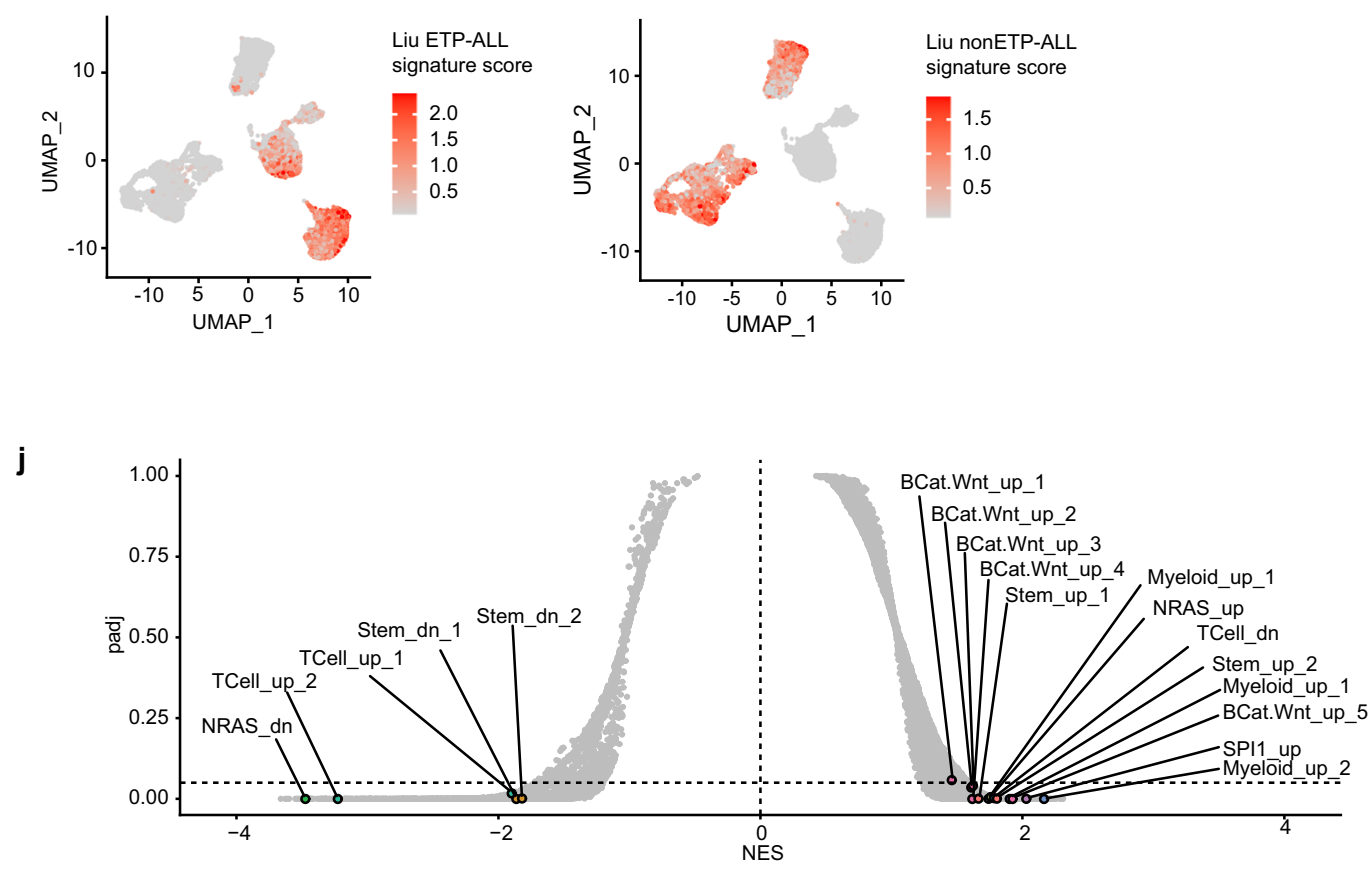

TCF7-SPI1 and NRAS(G12D) expression leads to aggressive T- TCF7-SPI1 with or without NRAS(G12D) within developing cell leukemia in a mouse bone marrow transplant model. We next sought to determine whether the combination of TCF7-SPI1 (TCF1-PU.1) and NRAS(G12D) cooperates in vivo to drive TALL. To this end, HSPCs isolated from the bone marrow of CD2Cre positive C57BL/6J mice were retrovirally transduced with the different lox66/71 vectors (Fig. 3b-d) to restrict expression of $\mathrm{T}$ cells (Fig. 4a). Wild-type recipient mice that received TCF7SPI1 only transduced cells did not develop leukemia within the 200 days post injection observation period. Recipient mice that received TCF7-SPI1 + NRAS(G12D) developed leukemia (median disease-free survival $=66$ days) with a similar latency to those that received NRAS(G12D) alone (median disease-free survival = 
Fig. 2 Single-cell RNA sequencing identifies that high SPI1 expression is associated with NRAS, stem cell, and Wnt/ $\boldsymbol{\beta}$-catenin signaling cell signatures. a-c UMAP plot of 11,620 malignant T-cells from 6 T-ALL samples with cells colored by their patient of origin (a), TCF7-SPI1 and TCF7-SPI1 + NRAS mutation status (b), level of SPI1 expression (c), the scalebar represents normalized expression values. d Heat map for all single cells across 6 T-ALL patients highlighting top 25 and bottom 25 genes correlated with SPI1 expression. e-g UMAP plot for expression levels of CD3D (e) CD1E (f) and, CD34 (g) highlighting differential expression between fusion-positive and fusion-negative T-ALL cases. $\mathbf{h}, \mathbf{i}$ UMAP plot of cells colored by the average expression of the ETP-ALL (h) or nonETP-ALL (i) signature derived from Liu et al. ${ }^{5}$, the scalebar represents averaged and scaled gene signature expression values. $\mathbf{j}$ Associated GSEA analysis pathways associated with SPI1 expression highlighting enrichment of stem, Wnt/ $\beta$-catenin, and myeloid signature. Full annotation and associated statistic of each pathway provided in Supplementary Data 8.

74 days). Both were confirmed acute leukemias with secondary recipients succumbing to leukemia with a significantly decreased latency (Fig. 4b).

Although NRAS(G12D) alone and TCF7-SPI1-P2A-NRAS (G12D) both gave rise to leukemia with a similar latency, a clear difference was observed in disease presentation and immunophenotype. Mice expressing NRAS(G12D) alone within developing $\mathrm{T}$ cells had an enlarged thymus but rarely had increased white blood cell (WBC) counts or leukemic invasion of the spleen. In contrast, mice expressing both NRAS(G12D) and TCF7-SPI1 presented with leukocytosis and splenomegaly but no enlarged thymus (Fig. 4c). This phenotype remained unchanged after secondary transplantation (Fig. 4d). The resulting leukemias were further phenotyped using flow cytometry with NRAS(G12D) leukemic thymic cells having a more differentiated CD4+/CD8+ immunophenotype and TCF7-SPI1 + NRAS(G12D) leukemic cells a CD4-/CD8-, (intracellular) CD3+ and CD117+ immature immunophenotype (Fig. 4e). Subsequently, an in vivo limiting dilution assay was performed to evaluate the frequency of leukemic stem cells (LSCs). This showed a significantly higher LSC frequency in the TCF7-SPI1-P2A-NRAS(G12D) mice (1/ $1243)$ compared to the NRAS(G12D) only condition (1/312092) (Supplementary Fig. 5).

TCF7-SPI1-induced immature leukemia requires upstream $\beta$ catenin activity. Given the identification of $\mathrm{Wnt} / \beta$-catenin signaling signatures in association with SPI1 expression in the single-cell data and the presence of a $\beta$-catenin binding domain at the $\mathrm{N}$-terminal region of the fusion protein, we next determined whether the $\mathrm{N}$-terminal $\beta$-catenin binding domain of TCF1 within the fusion protein is also important in leukemia development. TCF proteins are well known to be effectors of canonical Wnt signaling together with $\beta$-catenin ${ }^{27}$. Furthermore, $\beta$-catenin has previously been suggested to be critical for LSC self-renewal and more recently linked to maintaining LSC stemness and SPI1 expression $^{28-30}$. We, therefore, set up a new bone marrow transplant where we selectively removed the first 55 amino acids of TCF1 (TCF7) that comprise the $\beta$-catenin binding site (Fig. 5a). Similar to the leukemia that develops in NRAS(G12D) only mice (Fig. 4), recipient mice that received $\triangle \beta$ Cat-TCF7-SPI1 only had an enlarged thymus, limited splenic invasion, and a more mature CD4+/CD8+ immunophenotype with similar disease latency (median DFS $=67.5$ days) (Fig. 5b-d). This indicates that the deletion of the $\beta$-catenin binding domain abolished the action of the fusion leading to a NRAS(G12D) only disease.

To further determine whether loss of $\beta$-catenin binding was responsible for the altered disease presentation, we generated a series of GAL4 DNA binding domain fusion constructs, containing the N-terminal TCF7 part of the TCF7-SPI1 fusion or a truncated $\triangle \beta C$ at-TCF7, and performed luciferase reporter gene expression assays to assess transcriptional activity in HEK293T cells in the presence or absence of the GSK3 $\beta$ inhibitor CHIR-99021 to activate upstream Wnt signaling and increase $\beta$ catenin levels. The activation of $\mathrm{Wnt}$ signaling resulted in increased luciferase activity only for the full length but not for the $\triangle \beta C$ at-TCF7 construct (Fig. 5e, Supplementary Fig. 6a) and this was phenocopied in the melanoma Mel888 cell line, where $\beta$ catenin is constitutively active due to a S37F mutation ${ }^{31}$ (Fig. 5f). We observed a similar difference between TCF7-SPI1 and $\triangle \beta C$ atTCF7-SPI1 fusion constructs using Lambda B1 or Fes promotor luciferase reporter assays that both have endogenous SPI1 binding sites 32 (Supplementary Fig. 6b). The luciferase activity induced by full length SPI1 is lower than the activity induced by the fusion TCF7-SPI1 and is at a similar level as for the $\triangle \beta C$ atTCF7-SPI1 fusion (Fig. 5g). Taken together, these data demonstrate that the TCF7-SPI1 fusion induced immature T-ALL phenotype is driven in part by upstream $\beta$-catenin activity.

Genetic and small molecule antagonism of the TCF7-SPII fusion results in leukemia phenotype differentiation. To further characterize the function of the TCF7-SPI1 fusion in leukemic maintenance and survival, we undertook an inducible knockdown strategy using an all in one Tet-On vector system to express short hairpin RNAs (Fig. 6a) ${ }^{33}$. X09 PDX cells were transduced with constructs encoding either a shRNAmir targeting the fusion transcript (shSPI1_885) or control Renilla luciferase (shREN_713), sorted and expanded to reach $>90 \%$ mCHERRY positivity in vivo, then divided to receive either doxycycline or control chow at $0.1-1 \%$ human CD45 in the peripheral blood (Fig. 6b). At an end stage determined a priori, there was a $50 \%$ decrease in fusion expression but no decrease in leukemia burden in the peripheral blood or reduction of spleen weight upon doxycycline-induced knockdown of the fusion (Supplementary Fig. 7a, b). Strikingly, there was a significant increase in CD4 and CD8 and loss of CD117 (cKIT) cell surface expression with concomitant changes at the mRNA level (Fig. 6c, d, Supplementary Fig. 7b). This increased phenotypic differentiation was further supported by RNA-seq analysis, where knockdown of the fusion significantly decreased the ETP-ALL signatures (Fig. 6e, Supplementary Fig. 7c).

A global analysis of the differentially expressed genes to identify common regulatory elements using iCisTarget ${ }^{34}$ revealed that the significantly downregulated genes were highly enriched for SPI1 binding motif (Fig. 6f). To confirm the requirement of $\beta$ catenin for the oncogenic function of TCF7-SPI1 and to confirm the effect of fusion knockdown on differentiation, we made use of the small molecule $\beta$-catenin/TCF antagonist PKF $118-310^{27,35}$. PKF 118-310 completely abrogated the luciferase signal generated by TCF7-GAL4 or TCF7-SPI1 in Mel888 cells phenocopying the genetic removal of the $\beta$-catenin binding domain (Supplementary Fig. 7d). Moreover, similar to the iCisTarget results after knockdown of the fusion, treatment with PKF-118-310 in three TCF7-SPI1 fusion positive PDX samples also showed an enrichment of SPI1 motifs in the downregulated genes and decreased cKIT expression (Fig. 6f, Supplementary Fig. 7e, f). However, ex vivo treatment with PKF 118-310 did not show specific cytotoxicity toward fusion positive T-ALL cases compared to fusion-negative T-ALL cases (Supplementary Fig. $7 \mathrm{~g}$ ).

Taken together, these data demonstrate that the interaction of $\beta$-catenin with TCF1 (TCF7) is important for increasing the 
a

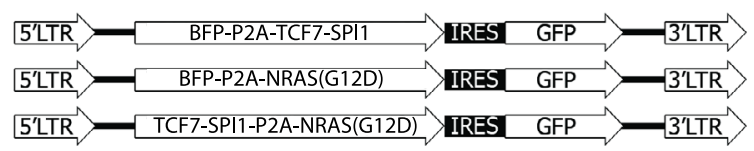

b

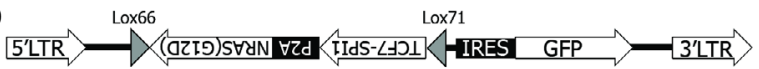

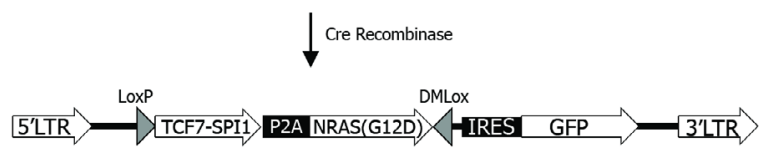
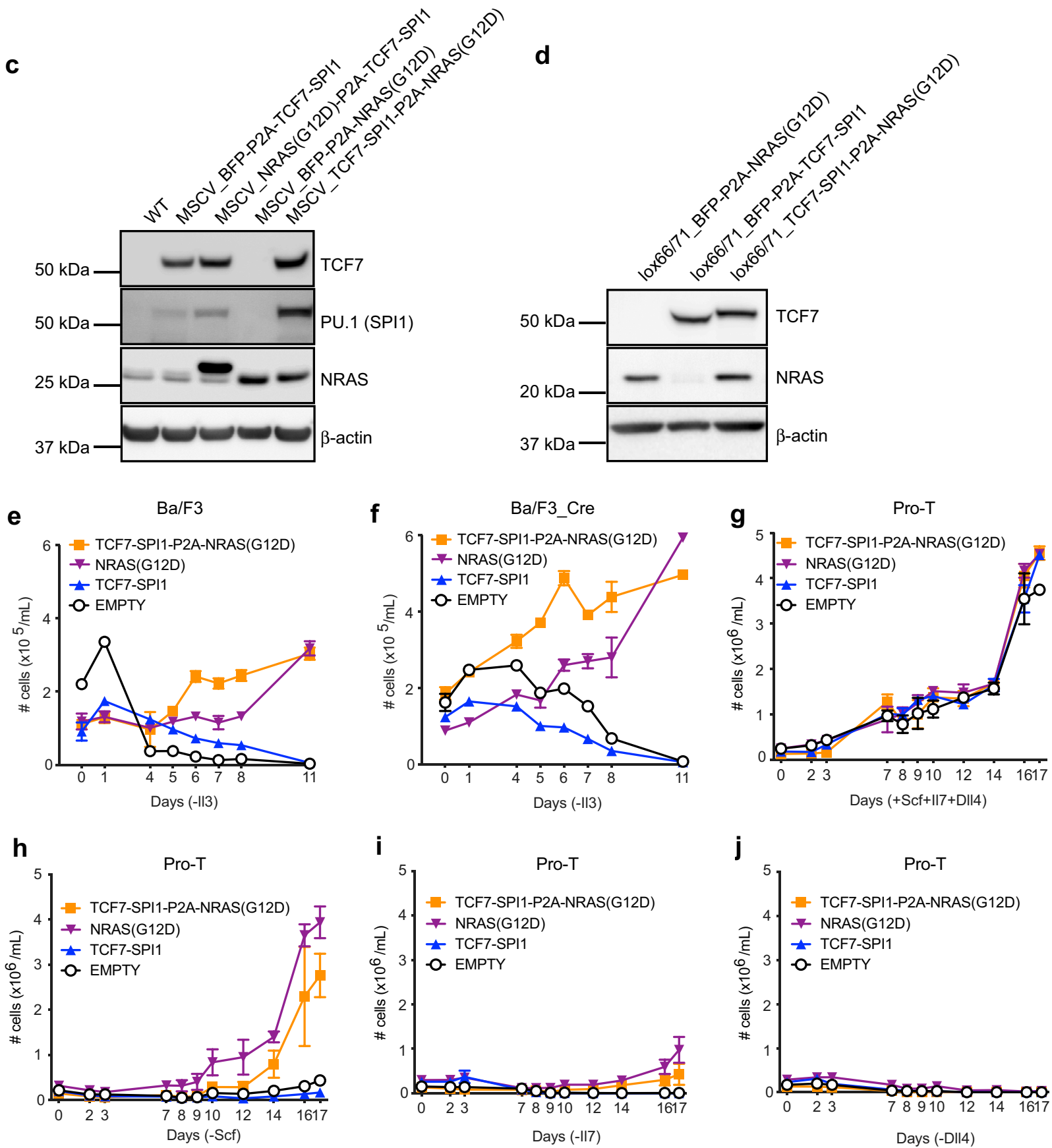

oncogenic function of the TCF7-SPI1 fusion and that its inhibition leads to downregulation of SPI1 targets and phenotypic differentiation of the leukemic cells.

\section{Discussion}

In 2017, Seki et al. described a distinct subset of pediatric T-ALL cases with dismal prognosis, characterized by an aberrant expression of SPI1 fusion genes. Seven out of 181 investigated patients carried translocations encompassing the SPI1 locus on chromosome 11 with TCF7 or STMN1 as typical fusion partners. Even though TCF7 appeared to be a stronger activator of PU.1 than STMN1, the authors were unable to induce T-cell leukemia in a mouse model by constitutively expressing TCF7-SPI1 on its own ${ }^{9}$. This suggests that additional proliferative and oncogenic signals are needed for leukemic transformation. In our center we identified a patient with a CD4/CD8 double negative T-ALL, characterized by the TCF7-SPI1 fusion in combination with a 
Fig. 3 The presence of the NRAS(G12D) mutation is necessary for in vitro transformation to cytokine-independent growth. a Constitutive retroviral vectors with either BFP-P2A-TCF7SPI1, BFP-P2A-NRAS(G12D) or TCF7-SPI1-P2A-NRAS(G12D) followed by an IRES-sequence and GFP. b Schematic representation of the inducible Cre-Lox system. The same constructs as in (a) were cloned in the antisense direction between 2 anti-parallel asymmetric Lox66/Lox71 sites. Upon expression of Cre-recombinase the sequence is flipped in a unidirectional manner. $\mathbf{c}$, $\mathbf{d}$ Western blot analysis showing expression of TCF7, SPI1, and NRAS for the different indicated constructs in Ba/F3 cells $(\mathbf{c})(n=1)$ or Cre-Ba/F3 cells with active Cre-recombinase (d) $(n=1)$. WT $=$ Wild-type Ba/F3 and used as a control lysate. The viral P2A sequences within the expression vector results in a $3^{\prime}$ in-frame 18 amino acid sequence tail on the first protein expressed accounting for the increased molecular weight for NRAS(G12D) and TCF7-SPI1 in (c) and (d), respectively. e Growth curve of $\mathrm{Ba} / \mathrm{F} 3$ cells transduced with the vectors illustrated in (a) or empty vector (white). $\mathbf{f}$ Growth curve with floxed constructs illustrated in (b) in Ba/F3-Cre cells alongside an empty vector control. $\mathbf{g}$-j Growth curves in pro-T cells after transduction with the indicated constructs and empty vector. Either no growth factors ( $\mathbf{g}), \operatorname{Scf}(\mathbf{h}), \| 17$ (i) or DII4 (j) were omitted. $\mathbf{e}-\mathbf{j}$ The number of cells is shown as a mean with standard deviation, $n=3$ independent experiments for each condition.

NRAS(G12D) mutation. The frequency of mutations in RAS signaling in pediatric T-ALL is estimated to approximate $15 \%$, but in the cohort of Seki et al., NRAS mutations were found in almost half of the cases with a SPI1 fusion ${ }^{9,36}$.

In this current work, we were able to demonstrate that the TCF7-SPI1 fusion can cooperate with NRAS(G12D) to induce an aggressive, CD4/CD8 double negative T-cell leukemia in vivo. Concordant with Seki et al., TCF7-SPI1 alone did not induce TALL in our bone marrow transplant model. Expressing NRAS (G12D) alone using our conditional retroviral expression system limiting NRAS(G12D) to developing T cells caused a mature Tcell leukemia/lymphoma characterized by thymus enlargement. Other research groups have also reported on the development of hematologic diseases such as myeloproliferative diseases and TALL when expressing oncogenic RAS mutations in mouse progenitor cells ${ }^{37,38}$. However, combining both oncogenic alterations in our conditional bone marrow transplant model resulted in an aggressive acute T-ALL characterized by hyperleukocytosis, splenomegaly and the absence of thymus enlargement. Although no difference in disease latency was observed, the altered disease presentation when both TCF7-SPI1 and NRAS(G12D) are expressed was also found to cause a significantly higher frequency of leukemic stem cells. The combination also resulted in a more immature immunophenotype than mutated NRAS alone with a double negative leukemia (CD4- and CD8-), similar to the immunophenotype of the patient X09. This suggests that the TCF7-SPI1 fusion provides a transcriptional program to block differentiation in the early thymic progenitor stage of development. Recently, it was shown that the TCF7-SPI1 fusion blocks differentiation of murine $\mathrm{T}$ cells in the DN3a stage $\mathrm{s}^{39}$.

This is also in part supported by our earlier findings ${ }^{40}$, where the order of mutational acquisition predicted that the translocation leading to the TCF7-SPI1 fusion occurred prior to acquisition of the NRAS mutation. Hence, the observed immature T-ALL phenotype in our conditional bone marrow transplant models is the result of the fusion blocking $\mathrm{T}$-cell differentiation and the NRAS(G12D) mutation providing the necessary proliferative signals for leukemia development. This model was also recently described for a TCF3-HLF fusion protein in B-ALL which cooperates with ETS factors and also leads to a block in differentiation ${ }^{41}$. Our data, therefore, posit that the observed cooccurrence of SPI1 fusions with RAS mutants is driven by a positive selective pressure in the development of T-ALL.

We also demonstrated that the TCF7-SPI1 fusion is dependent on $\beta$-catenin. Genetically, the TCF7-SPI1 construct lacking the $\beta$ catenin binding domain was sufficient to alter the disease presentation in vivo to be equivalent to NRAS(G12D) alone with a more mature CD4+/CD8+ immunophenotype. This was further demonstrated using PKF 118-310, which specifically inhibits the interaction between $\beta$-catenin and TCF1 and led to significant downregulation of SPI1 target genes in three separate TCF7-SPI1 fusion PDX cases.
In normal T-cell development, the controlled expression of the SPI1 transcription factor plays an important role in delaying the onset of T-cell commitment and possibly contributing to the expansion of early $\mathrm{T}$ cells ${ }^{42}$. In T-ALL, the mutually exclusive and ectopic expression of transcription factors such as TLX1, TLX3, and TAL1 is a key feature of T-ALL and often results from chromosomal translocations ${ }^{43}$. In this current work, we demonstrate that ectopic expression of SPI1 can occur via chromosomal translocations and a unique situation where $\beta$-catenin hijacks the SPI1 transcriptional program. This is in line with the recent finding that expression of SPI1 is important for maintaining the 'stemness' of T-ALL leukemic stem cells in a regulatory loop involving both HAVCR2 and $\beta$-catenin ${ }^{30}$. Given these findings, we speculate that the ectopic and deregulated expression of SPII may be a more general feature of immature T-ALL that either occurs by chromosomal translocations or potentially by cryptic mutations in cis-regulatory regions as has been recently described for TAL1 ${ }^{44}$.

Targeting the fusion in vivo with an inducible knockdown system did not have a profound effect on leukemia burden. However, decreasing expression of the fusion did lead to increased differentiation, providing evidence the differentiation block observed in murine systems is directly relevant to clinical samples in maintaining leukemic cells in an immature state. Induction of fusion knockdown at an earlier time point before reaching $1 \%$ human CD45 in the peripheral blood might have resulted in an eventual exhaustion of T-ALL through differentiation even in the presence of the NRAS mutation but could not be answered in our in vivo experiment conditions where our ethical end point was predetermined. Of interest is the comparison with acute promyelocytic leukemia (APL), which was the paradigm of differentiation therapy. In recent years, it has become clearer that by targeting the $P M L-R A R \alpha$ fusion, other mechanisms, such as senescence, are also contributing to the clearance of APL cells ${ }^{45}$. In many other leukemias, e.g., IDH mutant AML, mutations can cause a differentiation block, but are dispensable for disease maintenance ${ }^{46}$. Here we show that such a therapy might be relevant for the first time in T-ALL upon the development of improved TCF- $\beta$-catenin interaction inhibitors that may also be able to eliminate leukemic stem cells. In those cases that also have NRAS mutation, combinatorial treatments might be required. Interestingly, during the revision of this manuscript, Gocho et al. identified T-ALL cases that are sensitive to dasatinib, some of which were positive for TCF7-SPI1 without a NRAS mutation ${ }^{39}$. What remains unclear is the precise mechanism by which mutated NRAS and the TCF7-SPI1 fusion cooperate to drive T-ALL. NRAS might exert its oncogenic function through the MAPK pathway. On the other hand, RAS is known to activate the PI3K/AKT pathway, thereby inhibiting GSK3 $\beta$ and thus activating $\beta$-catenin ${ }^{47}$. Independent of the mechanism, we do show for the first time that antagonism of the TCF/ $\beta$-catenin interaction is sufficient to induce differentiation, providing a 
a

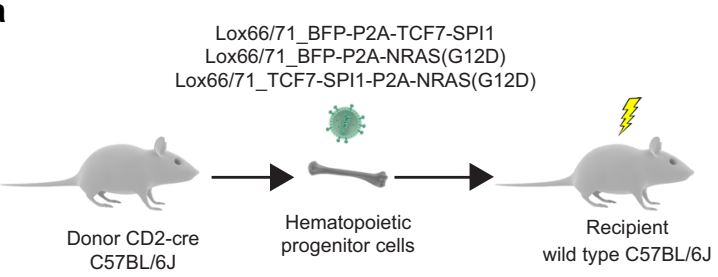

b

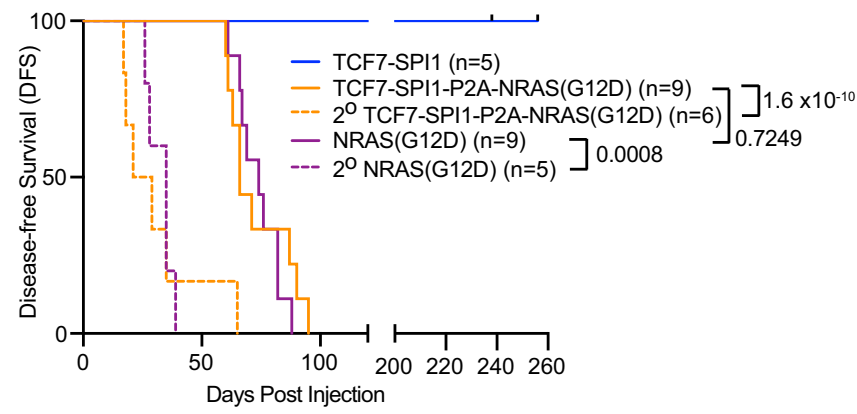

C

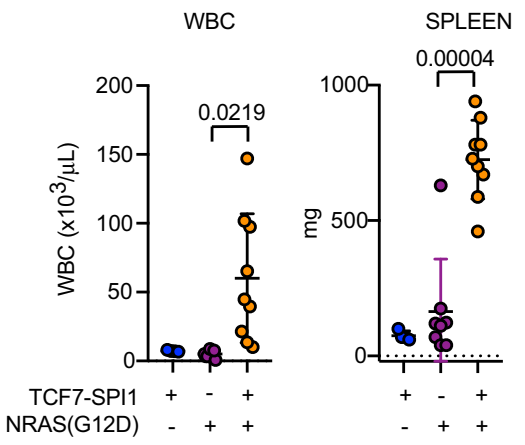

THYMUS

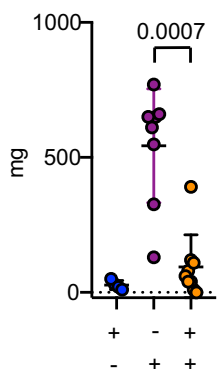

d

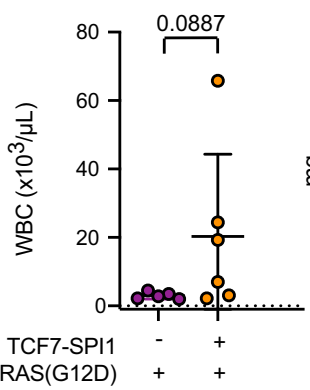

SPLEEN

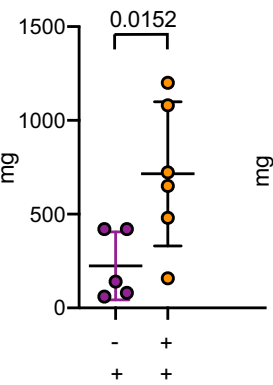

THYMUS

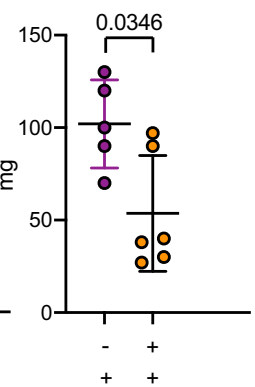

e

GFP+/-BFP positive cells

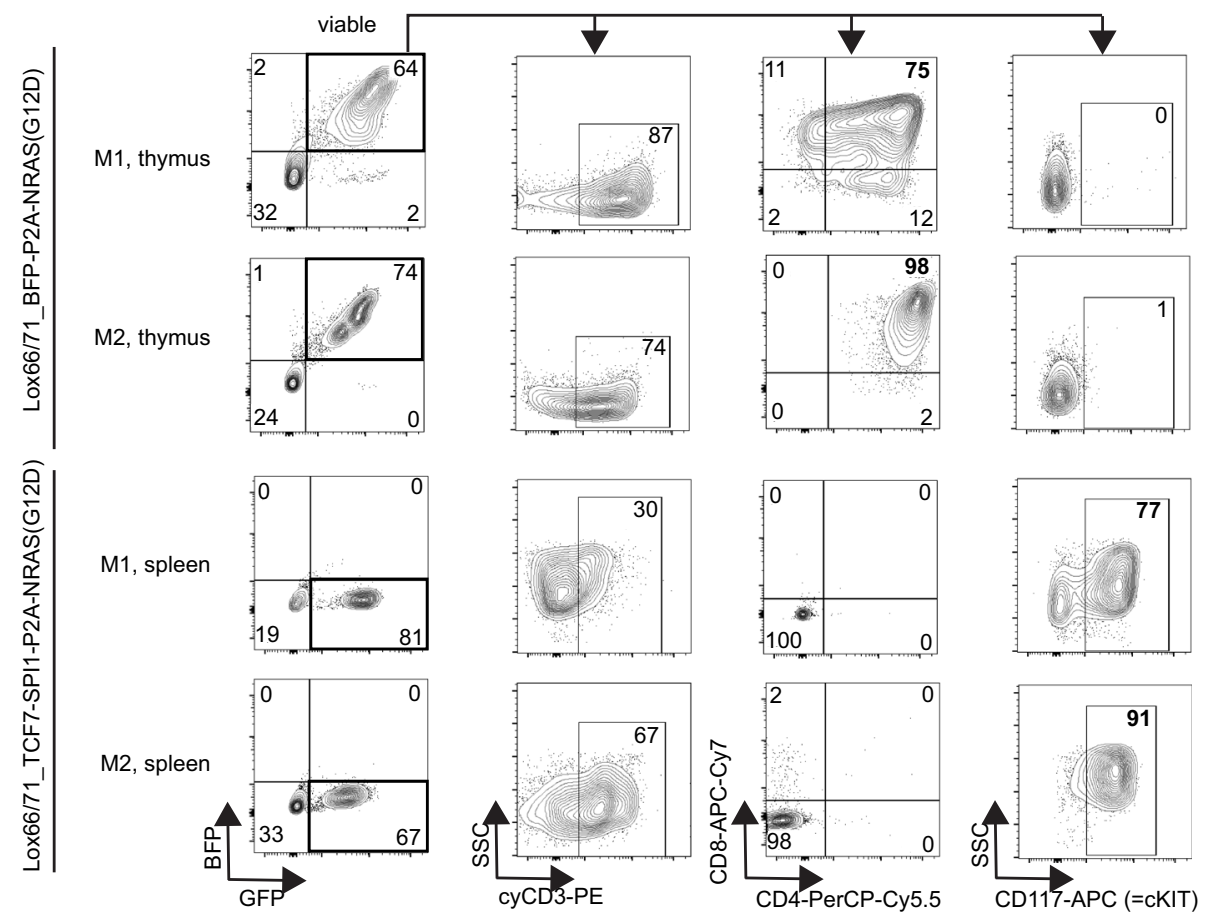

Fig. 4 Conditional co-expression of TCF7-SPI1 fusion and NRAS(G12D) within developing T cells cooperate to generate an immature T-cell acute lymphoblastic leukemia. a Schematic representation of the conditional bone marrow transplant (BMT) model. b Survival curve showing the disease-free survival with no significant difference in disease latency between NRAS only and TCF7-SPI1-P2A-NRAS $(p=0.7429)$. Secondary transplantations (dashed lines) have significantly shorter disease latencies ( $p=0.0008$ for NRAS only; $p=<0.0001$ for TCF7-SPI1-P2A-NRAS). Log-rank (Mantel-Cox) test. c White blood cell (WBC), spleen weight and thymus weight at end stage for the primary BMT. $n=4$ biologically independent animals for TCF7-SPI1, 8 for NRAS only (for the WBC graph $n=9$ ) and $n=9$ biologically independent animals for TCF7-SPI1 + NRAS in the three graphs. $\mathbf{d}$ Equivalent WBC, spleen, and thymus data for secondary transplantation. c, $\mathbf{d}$ Each point represents a different mouse, $n=5$ biologically independent animals for NRAS only and 6 for TCF7-SPI1 + NRAS; the mean is shown with standard deviation. $P$ values are indicated and were calculated by a one-way ANOVA with post hoc Dunnett T3 multiple comparisons test for (c) and a two-tailed Mann-Whitney test for (d). e Immunophenotyping of two different mice from (b) for each condition with spleen or thymus cells. Flow cytometry for cyCD3, CD4, CD8, CD117 are shown after gating on the viable and GFP+ cells. Distinguishing markers between the two conditions and their respective percentages are highlighted in red. 
a

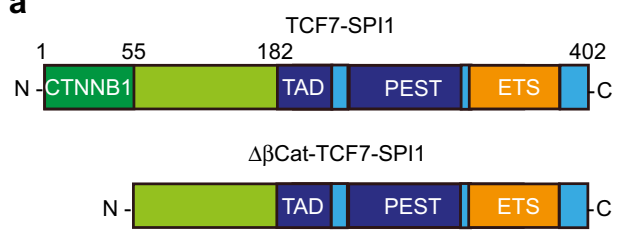

b

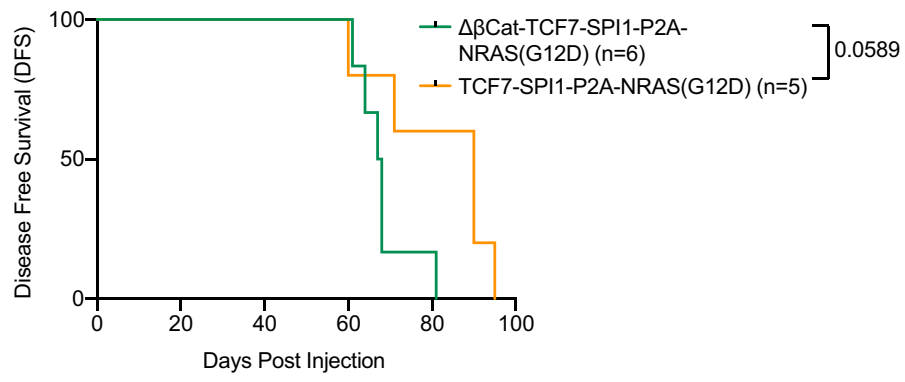

d
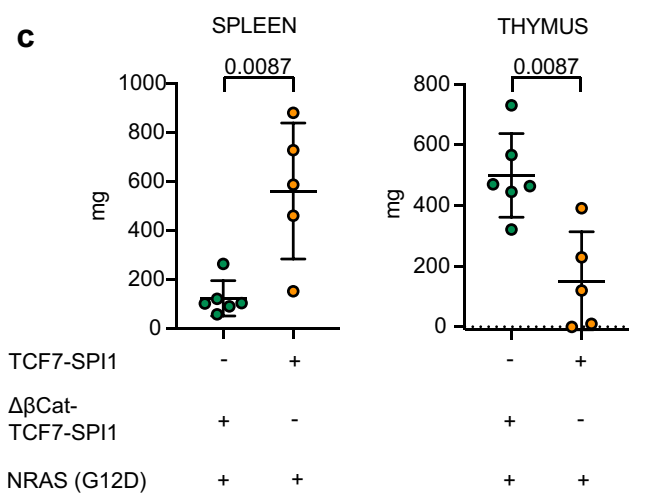

e

CHIR-99021

HEK293T

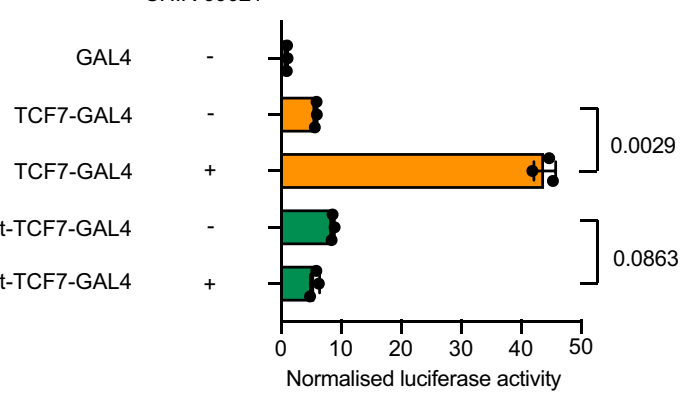

g

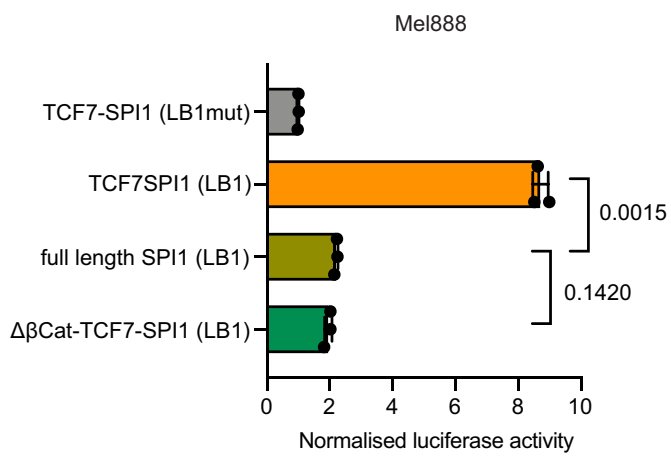

unique proof of principle that, in this instance, it is possible to directly target an oncogenic transcription factor.

In conclusion, we have shown that TCF7-SPI1 cooperates with NRAS (G12D) to drive an aggressive immature T-cell leukemia in vivo and that it is possible to target this fusion protein by inhibiting protein-protein interactions between TCF1 and $\beta$ catenin. Patients with the TCF7-SPI1 fusion could thus potentially benefit from an improved pharmacological targeting of the TCF/ $\beta$-catenin interaction.

\section{Methods}

Patient sample collection and storage. Fresh bone marrow and peripheral blood samples were collected from newly diagnosed ALL patients in the Pediatric Hemato-Oncology Department of the University Hospitals Leuven at time of 
Fig. 5 The $\mathbf{N}$-terminal $\beta$-catenin binding site of the TCF1-PU.1 fusion protein is essential for transcriptional activity and ETP-ALL development in vivo. a Schematic representation of the protein domains resulting from the entire TCF7-SPI1 fusion gene (above) or without (below) the $\beta$-catenin binding domain (CTNNB1) at the N-terminus (first 55 amino acids). b Kaplan-Meier curve for disease-free survival with the indicated inducible constructs. Logrank (Mantel-Cox) test, $p=0.0589$. c Spleen and thymus weights at end stage for mice in (b). Dots represent different mice ( $n=6$ biologically independent animals for $\triangle \beta$ Cat-TCF7-SPI1 and 5 for TCF7-SPI1); the mean is shown with standard deviation. $P$ values are indicated and were calculated by a two-tailed Mann-Whitney test. d Flow cytometry staining of a thymus sample of a $\Delta \beta$ Cat-TCF7-SPI1-P2A-NRAS(G12D) mouse. Staining for CD4 and CD8 is shown after gating on GFP-positive cells. e- $\mathbf{g}$ Dual luciferase assay as described in "Methods". e HEK293T cells with GAL4 constructs. Overnight treatment with CHIR-99021 at a dose of $1 \mu \mathrm{M}$. $\mathbf{f}$ Mel888 cells with GAL4 constructs. $\mathbf{g}$ Mel888 cells with SPI1 reporter gene containing the LB1 promotor or mutant. Results were normalized to the average GAL4 signal. $P$ values $(\mathbf{e}-\mathbf{g})$ are indicated, one-way ANOVA with post hoc Dunnett T3 multiple comparisons test. Mean with standard deviation is shown. Dots represent different samples, $n=3$ independent experiments per condition. Repeat experiments of (e) and (f) are shown in Supplementary Fig. 5.

diagnosis and at remission on protocol S57176 approved by the Ethical Committee University Leuven. Written informed consent was obtained from every patient in accordance with the Declaration of Helsinki. Mononuclear cells were isolated using Ficoll-Paque and viably frozen in $95 \%$ fetal calf serum and 5\% DMSO, or immediately injected into immune deficient NOD.Cg-Prkdcscid Il2rgtm 1 Wjl/SzJ (NSG) mice for further leukemic expansion.

Whole-genome and transcriptome sequencing. DNA and RNA were extracted from the diagnostic sample (Maxwell, Promega) and prepared for sequencing using the KAPA Hyper Prep kit (Illumina). Libraries were sequenced on a HiSeq2500 with $125 \mathrm{bp}$ paired-end reads (Illumina). Poor quality reads were trimmed using fastq-MCF (ea-utils) $)^{9}$ and the quality was checked with fastQC (v0.11.9) (http:// www.bioinformatics.babraham.ac.uk/projects/fastqc) before alignment with Bowtie2 (v2.3.5.1) (DNA) ${ }^{48}$ or Tophat2 (v2.1.1) (RNA) ${ }^{49}$ to human reference genome GRCh37. Duplicates were removed using Picard and we applied VarScan (v2.4.4) $)^{50}$ to identify true somatic mutations and indels between the leukemia and remission samples. We included somatic mutations with a VAF of at least $5 \%$ in the tumor sample, in order to allow detection of mutations in minor subclones. In addition, the relative difference between the VAF in the tumor and the VAF in the remission sample was required to be minimum $20 \%$ and significant based on Fisher's exact test, and the VAF at remission was $<5 \%{ }^{40}$. Chromosomal translocations were determined in the DNA by BreakDancer (v1.1_20100719) and we used FusionCatcher (v1.33) to detect gene fusions in the RNA data. Chromosomal breakpoints and mutations of interest were visually confirmed in IGV (v2.8.2) and verified by PCR and Sanger sequencing.

Nanopore sequencing of primary X09 sample. Viably frozen diagnostic peripheral blood cells were thawed at $37^{\circ} \mathrm{C}$ followed by suspension in phosphatebuffered saline (PBS) supplemented with $10 \%$ fetal calf serum. After the cells were washed, mRNA was extracted using the Fast track MAG - maxi mRNA isolation kit (Thermo Fisher Scientific) and prepared for sequencing on the GridION X5 (Oxford Nanopore Technologies) according to the manufacturer's protocol. The sequencing data was then mapped to the human reference genome (hg38) using Minimap2 (v2.17-r941), after which it was inspected with the IGV (v2.8.2) software ${ }^{51}$.

Flow cytometry. Cells were washed with PBS and prepared at a concentration of $1 \times 10^{6} / \mathrm{mL}$ before staining and analysis on the MACSQuant VYB (Miltenyi Biotec), Fortessa (BD) or FACS Verse (BD). For the primary/xenograft and mouse cells, the antibodies listed in the supplementary table were used (Supplementary Data 10). FlowJo (v10.6.1; Tree Star) software was used to analyze the data. Full immunophenotypic profile of the patient samples can be found in Supplementary Data $1-3$.

Cell culture and retroviral transduction. 293T cells were cultured in RPMI medium supplemented with $10 \%$ fetal bovine serum (FBS) and transfected using GeneJuice (Merck Millipore). Ba/F3 cells were cultured in RPMI medium with $10 \%$ FBS in the presence of $1 \mathrm{ng} / \mathrm{mL}$ interleukin-3 (Il3). Ba/F3-Cre cells were generated through retroviral transduction with MSCV-Cre recombinase-Puro vector and selection with $1 \mu \mathrm{g} / \mathrm{mL}$ puromycin (LifeTechnologies) addition in cell culture media. After transduction with retroviral vectors, cell transformation was assessed in the absence of Il3 growth factor. Pro-T cell cultures were established from C57BL/6 mice by extracting lineage negative bone marrow cells and growing them on DLL4-coated plates. Before coating with DLL4 (in-house production at VIB Protein Core), the non-treated tissue culture plates (BD Falcon \#734-0949) are coated with anti-Fc (Abcam, ab1927). These cells are kept in culture in RPMI medium with $20 \%$ FBS, Il7 $20 \mathrm{ng} / \mathrm{mL}$ (PeproTech), Scf $20 \mathrm{ng} / \mathrm{mL}$ (PeproTech) and Primocin $100 \mu \mathrm{g} / \mathrm{mL}$ (Invivogen). Cell transformation was assessed in the absence of the different growth factors ${ }^{52,53}$.

Western blot analysis. Cells were lysed in cold lysis buffer, containing $5 \mathrm{mM}$ $\mathrm{NA}_{3} \mathrm{VO}_{4}$ and Complete (Roche), before separation of the proteins on NuPAGE
NOVEX Bis-Tris 4-12\% gels (Invitrogen). Analysis was performed with antibodies against NRAS (Abcam, Ab55391), TCF1 (Thermo Fisher Scientific, MA5-14965), PU.1 (Abcam-76542), and $\beta$-actin (Sigma-Aldrich). Bands were visualized using ImageQuant LAS-4000 (GE Healthcare).

Bone marrow transplant experiments. Lineage negative cells were isolated from the bone marrow of 6-to-8-weeks-old CD2-Cre C57BL/6J mice and transduced with retroviral vectors containing constructs encoding the TCF7-SPI1 fusion and/ or NRAS (G12D) mutant together with GFP downstream of an IRES. 0.5 to $1.5 \times$ $10^{6}$ transduced cells were injected into the tail veins of irradiated C57BL/6 J female recipients. Blood samples were taken at regular time points and analyzed on a Vet ABC Hematology Analyzer (SCIL) and flow cytometer (MACSQuant ${ }^{\circledR}$ VYB, Miltenyi Biotec) to determine the evolution in white blood cell and GFP $+(\mathrm{BFP}+)$ cell counts respectively. Mice were sacrificed when the white blood cell counts exceeded $20000 / \mu \mathrm{L}$ and/or ethical end point criteria were reached. Animal experiments were approved by the Ethical committee on animal experimentation of KU Leuven.

In vivo inducible knockdown mouse model. The LT3GECIR vector was provided by Prof. Dr. Johannes Zuber ${ }^{33}$ and shRNAmir sequences targeting Renilla Luciferase (Ren713) or human SPI1 (SPI1_885) were cloned into EcoRI/XhoI sites (Supplementary Data 11). Lentivirus was generated in 293FT cells and then transduced into X09 patient derived xenograft (PDX) cells for $24 \mathrm{~h}$ prior to injection into NRG immunocompromised mice (NOD.Cg-Ragltm1Mom Il2rgtm1Wjl/SzJArc) for expansion. Transduced cells were isolated from mice with mCHERRY positive cells sorted (FACS Aria III, BD) and enriched through multiple rounds of in vivo expansion until $>90 \%$ of human CD45+ cells were mCHERRY + . One million mCHERRY positive transduced X09 PDX cells expressing either LT3GECIR_shREN_713 or LT3GECIR_shSPI1_885 were then inoculated into $12 \mathrm{NRG}$ mice per group via tail vein injection. When mice reached $0.1-1 \%$ human CD45 in the peripheral blood, mice were randomly divided to receive either standard or Dox-impregnated chow (Doxycycline $=600 \mathrm{mg} / \mathrm{kg}$; Specialty Feeds, Western Australia). Leukemia burden was monitored via weekly peripheral blood sampling until the mice reach a defined end point a priori of $\sim 20 \%$ human CD45 positive cells in the blood. Authority to undertake animal work was approved by the Animal Care and Ethics Committee, University of New South Wales (Approval 20/6B)

Limiting dilution assay. A secondary transplantation was performed with decreasing numbers of leukemic cells for both TCF7SPI1 + NRAS(G12D) and NRAS(G12D) only: $2 \times 10^{6}, 2 \times 10^{5}$ cells, $2 \times 10^{4}$ cells and $2 \times 10^{3}$ cells. For each condition 5 WT C57BL/6 mice were injected after irradiation with 2,5 Gy, in total 40 mice were used. Disease was defined as a GFP percentage in the blood of $10 \%$.

Dual luciferase assay. HEK293T or Mel888 cells were transfected in a 12-well plate with $500 \mathrm{ng}$ ( $3 \mu \mathrm{g}$ for Mel888) of pGL 4.35 (Promega) and different GAL4 constructs (GAL4, TCF7-GAL4 and $\triangle \beta$ Cat-TCF7-GAL4), and $25 \mathrm{ng}$ of RL-TK (75 $\mathrm{ng}$ for Mel888). After $24 \mathrm{~h}$ the cells were lysed with Passive Lysis Buffer and light intensity was measured on a Victor multilabel plate reader (PerkinElmer) using the Promega Dual-Luciferase ${ }^{\circledast}$ Reporter Assay System (catalog number: E1910) according to the manufacturer's instructions. For each well, firefly luciferase activity was normalized to Renilla activity. In the experiments with PKF 118-310 the results were normalized to either GAL4 or the mutant Lambda B1 reporter because of interference of PKF with Renilla luciferase activity. Previously, other drugs have also been reported to interfere with luciferase activity ${ }^{54}$. The used inhibitors were CHIR-99021 (Selleck Chem-S1263) and PKF 118-310 (SigmaAldrich-K4394). The Mel888 cells were kindly provided by the lab of prof. L. Larue. The SPI1 luciferase reporter genes were kindly provided by the lab of prof. O. Bernard.

qPCR. Total RNA was extracted using using NuclepSpin RNA Plus columns (Macherey Nagel) and $500 \mathrm{ng}$ reverse transcribed into cDNA using random 
a

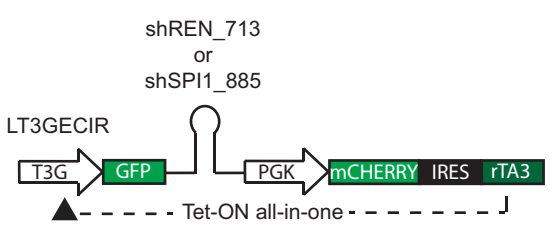

C LT3GECIR_ShREN_713

(Event $=$ Day 21$)$

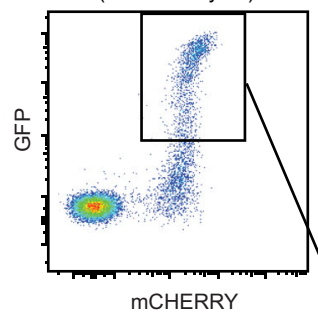

LT3GECIR shSPI1 885

MCHERRY b

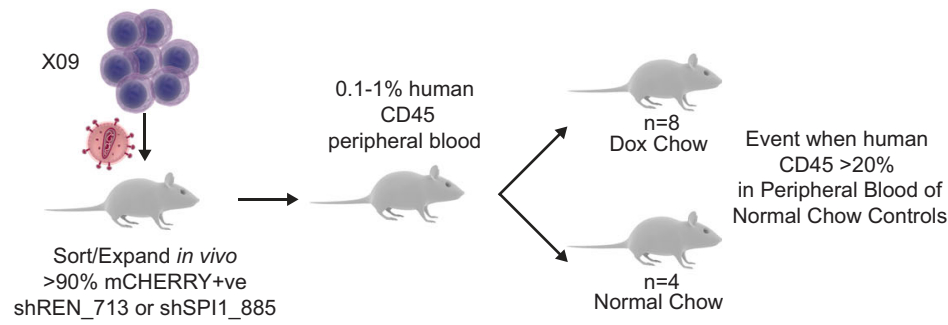

ShREN 713 or ShSPI1 885

d $($ Event $=$ Day 14$)$
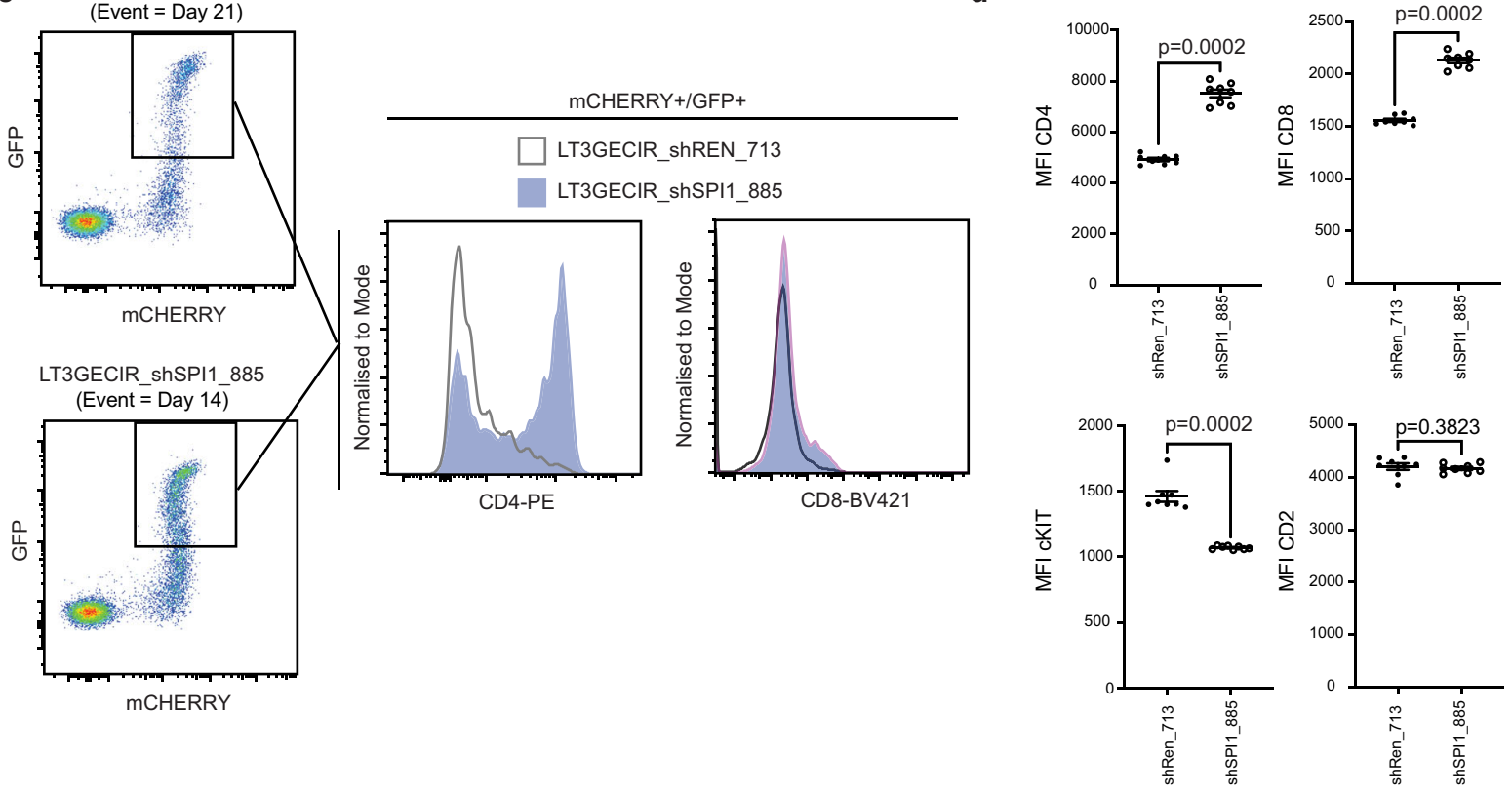

e

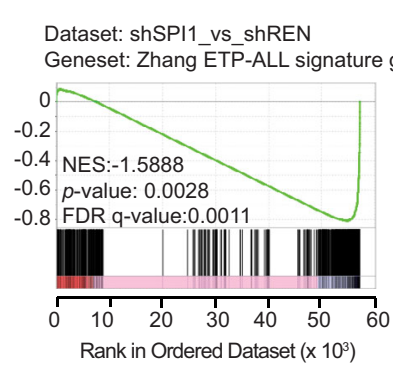

Dataset: shSPI1 vs shREN

Geneset: Liu ETP-ALL signature genes

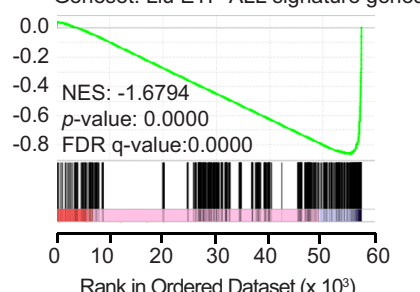

Rank in Ordered Dataset $\left(\times 10^{3}\right)$ f
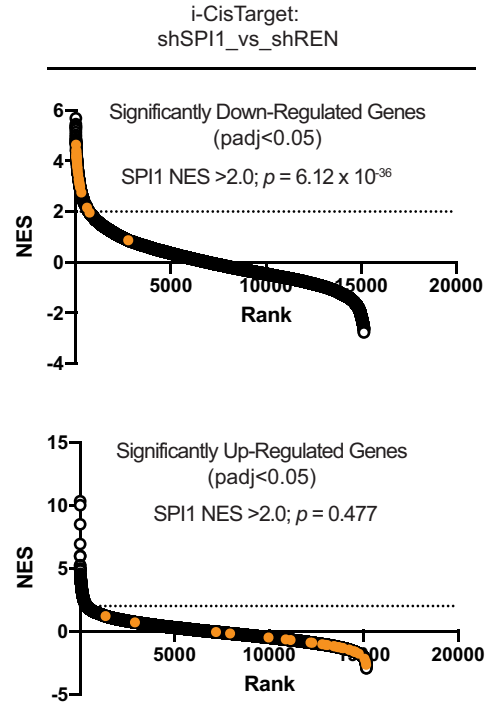

i-CisTarget: X09 (PDX) 2 M PKF118-310_vs_DMSO
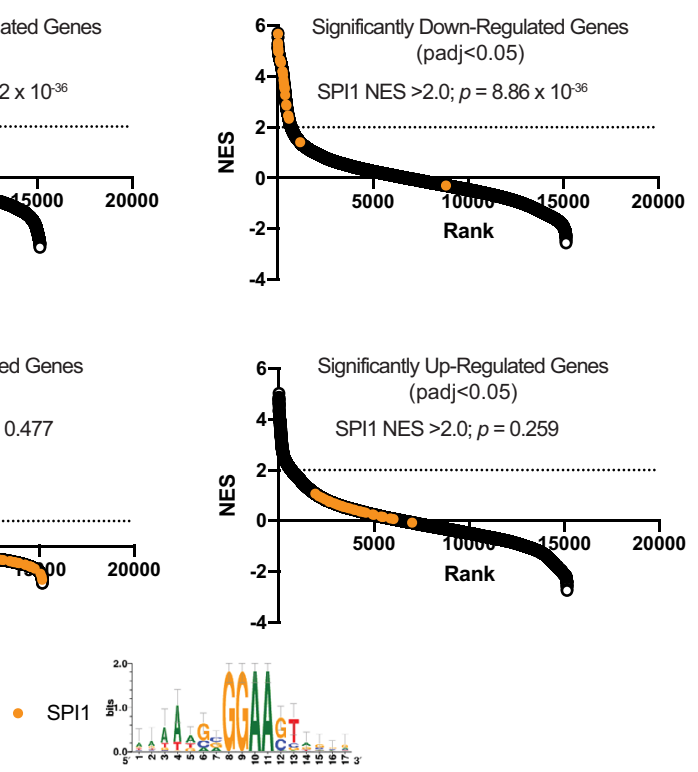

primers according to the manufacturer's protocol (GoScript cDNA Synthesis Kit, Promega). Real-time quantitative was performed using GoScript SYBR master mix kit (Promega) with the QuantStudio 3 PCR system.

(Applied Biosystem). Quality control, primer efficiency, and data analysis were carried out using qbase+ software (v3.2; Biogazelle). All gene expression was normalized using two housekeeping reference genes. The primer sequences for the qPCR experiments in Supplementary Fig. 7 can be found in Supplementary Data 12.
Dose-response curve. Leukemic cells were seeded into a 96-well plate at 300,000 cells/mL and the drug was added using a D300e digital dispenser (Tecan) in an increasing dose. After $24 \mathrm{~h}$, cell proliferation was measured using the ATPlite luminescence system on a Victor multilabel plate reader (PerkinElmer). Results were normalized to the DMSO condition. Results were analyzed with a non-linear regression. The analysis of the J188 PDX mouse was undertaken independently by the lab of Itaru Kato following the same protocol. 
Fig. 6 Genetic and small molecule antagonism of the TCF7-SPI1 fusion results in leukemia phenotype differentiation. a Schematic representation of the shRNAmir in the LT3GECIR vector. b Schematic representation of the in vivo inducible knockdown mouse model. Mice received doxycycline or normal chow. c FACS plots for a shREN_713 (above) and a shSPI_885 mouse (below). Staining for CD4-PE (left) and CD8-BV421 (right) is shown after gating on GFP and mCHERRY positive cells from peripheral blood, shREN_713 is represented in white and shSPI_885 in purple. d Mean fluorescence intensity (MFI) of CD4, CD8, cKIT, and CD2 GFP+/mCHERRY + peripheral blood is shown in mice with shREN_713 $(n=8)$ or shSPI_885 ( $n=8)$. Dots represent different mice. $P$ values are indicated and were analyzed with a two-tailed unpaired Mann-Whitney test. Mean is shown with standard error of the mean. e RNA-seq gene expression analysis in mice with shSPI1_885 versus shREN_713. GSEA using the Zhang and Liu ETP-ALL signature gene sets. Normalized enrichments scores, $p$ values, and FDR $q$ values are indicated. $\mathbf{f}$ iCisTarget motif analysis in mice with shSPI1_885 versus shREN_713 (left) or after $24 \mathrm{~h}$ ex vivo treatment with PKF 118-310 versus DMSO (right). Motifs in both down- (above) and upregulated genes (below) were ranked according to their respective normalized enrichment scores. SPI1 motifs are indicated in orange. $P$ values are indicated and were calculated with a hypergeometric distribution. All results can be found in Supplementary Data 15-22.

Bulk RNA-seq analysis of patient cohorts. RNA-seq data were retrieved for 123 T-ALL patients from the National Bioscience Database Center (NBDC) of the Japan Science and Technology Agency (JST), under the accession JGAS00000000090 and approved by the University of New South Wales institutional review board human ethics committee (HREAP HC200558). RNA-seq and patient-related data for two T-ALL patients (SJTALL03263, SJTALL031201) were obtained from St. Jude Hospital and approved by the University of New South Wales institutional review board human ethics committee (HREAP HC200562). Patients and/or their guardians for the samples SJTALL030263_D1 and SJT031201_D1 obtained from the St. Jude Biorepository, provided written informed consent in accordance with the Declaration of Helsinki. RNA-seq data previously generated in De Bie et al. ${ }^{40}$ for 4 T-ALL patients (X09, XB37, XB41, XB47) were also utilized. Paired-end reads were first trimmed to remove Illumina sequencing adapters and then filtered to remove poor quality or short read pairs $(<20$ base pair length, Phred33 quality score $<20)$ using TrimGalore (v0.6.6). Read pairs were then aligned to the human genome (GRCh38) using STAR (v.2.7.5c) $)^{55}$ in two-pass mode, whilst also retaining junction/chimeric reads for later fusion transcript detection. Aligned reads were then counted using HTSeq (v0.12.4) ${ }^{56}$ using GENCODE (v22) gene annotations. Gene-level read counts were additionally normalized to transcripts per million (TPM) using a custom R script (zenodo repository DOI: 10.5281/zenodo.4756105). Fusion transcripts were then detected using arriba (v1.2.0) ${ }^{57}$ with previously generated STAR outputs. Differential gene expression analyses were conducted between patient sub-groups using the DeSeq2 $\mathrm{R}$ package $(\mathrm{v} 1.22 .0)^{58}$, where differentially expressed genes were considered as those genes with a $\log _{2}$ foldchange $>1$ or $<-1$ and Benjamini-Hochberg adjusted $p$ value $<0.05$ (Supplementary Data 13). Gene set enrichment analyses (GSEA) were conducted using the fgsea R package (v1.14.0) ${ }^{59}$ against the molecular signatures database (v7.2), where input genes were ranked using -sign( $\log _{2}$ foldchange) ${ }^{*} \log _{10}$ (adjusted p-value). ETP-ALL and T-ALL gene signatures were derived from previous differential gene expression analyses in Zhang et al. ${ }^{25}$, where those genes that had fold change $>2$ and adjusted $p$-value $<0.05$ were considered to be part of the ETP-ALL signature and those genes with fold change $<-2$ and adjusted $p$ value $<0.05$ comprised the T-ALL signature (Supplementary Data 7 and 13). Gene signatures were calculated in each patient by first applying $\mathrm{z}$-score scaling across signature genes, then taking the signature mean, and finally rescaling the signature means across all patients. ETP-ALL and nonETP-ALL gene signatures were also constructed by first performing differential gene expression analysis between samples with an ETP status of "ETP" $(n=19)$ and "nonETP" $(n=146)$ with the DeSeq2 R package $(v 1.22 .0)^{58}$ using read counts deposited by Liu et al. ${ }^{5}$. Differentially expressed genes that had a fold change $>2$ and adjusted $p$-value $<0.05$ were considered to be part of the ETP-ALL signature and those genes with a fold change $<-2$ and adjusted $p$-value $<0.05$ comprised the nonETP-ALL signature (Supplementary Data 6). Gene signatures for each patient were calculated as described above.

Mutational analyses. Somatic mutations previously identified by targeted sequencing in Seki et al. were first obtained. Somatic mutations in the remaining 6 patients were derived from WGS data and annotated using the ensemble variant effect predictor (VEP) (Ensembl release 103) ${ }^{60}$. Only those mutations previously assessed in the targeted sequencing by Seki et al. were retained for association testing.

\section{Bulk RNA-seq for PKF 118-310 treated and inducible knockdown mouse} samples. For differential gene expression analyses RNA was collected using NuclepSpin RNA Plus columns (Macherey Nagel) from triplicate conditions $(2 \mu \mathrm{M}$ of PKF 118-310 or DMSO treated PDX cells and sorted mCHERRY+GFP+ blasts isolated from the spleen for the Dox-inducible short hairpin RNA knockdown shREN_713 and shSPI1_885). The X09 PKF 118-310 treated RNA was prepared at the Genomics Core of KU Leuven and $3^{\prime}$ end RNA sequencing was performed (QuantSeq). The St. Jude SJTALL03263_D1 and SJTALL031201_D1 and inducible knockdown RNA samples were polyA-enriched and sequenced at BGI Genomics (Hong Kong) using DNBSEQ platform at a depth of 20 million read pairs per sample (PE100). After cleaning the data with fastq-mcf and a quality control with FastQC (v0.11.9), the resulting reads were mapped with HISAT2 (v2.1.0) to the human reference genome (GRCh38) and the abundance of reads per gene was determined with HTSeq-count. Differential gene expression was performed with the R package DESeq2 (v1.22.0),. Enrichment of motifs, based on a database of position weight matrices (PWMs), in the regulatory elements of the significantly up and downregulated genes (padj $<0.05$ ) were determined with iCisTarget $\left(\right.$ v2015) ${ }^{34}$. Hockeystick plots based on the normalized enrichment scores (NES) were constructed, highlighting the PWMs related to SPI1. To determine the gene set enrichment of the ETP and T-ALL signature signatures in the ranked lists of differential genes the GSEA software of the Broad Institute was used.

Single-cell RNA sequencing data analysis. Bone marrow samples from four $\mathrm{T}$ ALL patients (X09, XB37, XB41, XB47) were previously profiled using the $10 \times$ Genomics Chromium ${ }^{\mathrm{TM}}$ 3'v2 single-cell gene expression platform $^{40}$. In addition, samples from two T-ALL patients (SJTALL030263, SJTALL031201) were sourced from the St. Jude Biorepository and profiled using 10x Genomics Chromium ${ }^{\mathrm{TM}}$ 3'v3 chemistry. Reads were demultiplexed and aligned to the human reference genome (GRCh38), followed by unique molecular identifier (UMI) counting using the CellRanger v3.0.2 software suite ${ }^{61}$. Raw UMI count matrices were used as input for the Seuratv3 $\mathrm{R}$ package (v3.2.3) for downstream processing of the data ${ }^{62,63}$. In droplet-based scRNA-seq methods, droplets can often contain ambient RNA and be misinterpreted as a cell, therefore we eliminated these putative empty droplets using the DropletUtils package (v1.4.3), set at a false discovery rate (FDR) of 0.01 $(1 \%)^{64}$. Furthermore, we filtered out "poor quality" cells that contained either; high proportions of ribosomal reads $(>0.6)$, high proportions of mitochondrial reads $(>0.1)$ or a low number of expressed genes $(<200)^{65}$. All cells were then integrated and batch-corrected using sctransform whilst regressing out features in the data associated with technical variation, such as; the number of genes expressed, the number of UMI counts, mitochondrial read proportions, ribosomal read proportions, and estimated phases of the cell cycle ${ }^{66}$. In addition, the Harmony package (v1.0) ${ }^{67}$ was used to predict a shared embedding that accounted for any batch variation that arose due to the varying v2 and v3 $10 \times$ Genomics Chromium ${ }^{\mathrm{TM}} 3^{\prime}$ chemistry of samples. Harmony was used for initial cell type classification of malignant and non-malignant cell types but after removal of non-malignant cells, all embeddings were created using standard principal component analysis (pca) and uniform manifold approximation and projection (UMAP) based methods without Harmony. Principle components with the highest variance were used to semi-supervise cell clustering using the Louvain algorithm ${ }^{62,63}$, wherein the optimal clustering resolution was determined using the clustree R package $(\mathrm{v} 0.4 .1)^{68}$. Dimensional reduction was then performed using UMAP to visualize transcriptomic variation among single cells ${ }^{69,70}$. SPI1 expression correlations were computed using median-scaled log-transformed \& normalized gene expression values from the "data" slot of the Seurat object. Correlation estimate was calculated according to the Pearson correlation coefficient. Expression heatmaps were generated for highly correlated and anti-correlated genes and visualized using the ComplexHeatmap package $(\mathrm{v} 2.0 .0)^{71}$. Gene-set enrichment analysis was undertaken on a dataset ranked according the SPI1 Pearson correlation coefficient, and enrichment statistics were evaluated for gene-sets of the Molecular Signatures database (MSigdb v7.4) using the fgsea package (v1.14.0) ${ }^{59,72}$. Gene signature expression scores of Zhang et al. ${ }^{25}$ and Liu et al..$^{5}$ signatures were computed for each cell using the AddModuleScore function of the Seuratv3 $\mathrm{R}$ package ${ }^{22,63}$ and scores were then re-scaled using z-score normalization. Processed single-cell RNAseq data of the human fetal thymic cell atlas were directly retrieved from the zenodo data repository under the accession 10.5281/zenodo.3572422. These data contained normalized count matrices, embeddings, and cell type annotations made in the original study ${ }^{26}$. Cells corresponding to either "T" or "innate T" cell types in "level_1" meta data annotations as well as early thymic progenitor (ETP) cells were subsetted. Gene signature expression scores were computed as described above. Two-sided pairwise $t$ tests were performed between cell groups, followed by 
Benjamini-Hochberg (BH) adjustment of resultant $\mathrm{p}$ values to correct for multiple hypothesis testing.

Amplification of the TCF7-SPI1 fusion from 10x cDNA libraries. A total of 0.1 ng of cDNA from 10x cDNA libraries for SJTALL031201_D1 was amplified using $2 \times$ KAPA HiFi HotStart ReadyMix and $0.4 \mu$ M Biotinylated TCF7 Exon 4 forward primer and $0.4 \mu \mathrm{M}$ Read 1 reverse primer (Supplementary Data 14) using 1 cycle of initial denaturation $95^{\circ} \mathrm{C}$ for $5 \mathrm{~min}$, followed by 15 cycles of $98^{\circ} \mathrm{C}$ denaturation for $20 \mathrm{~s}, 63^{\circ} \mathrm{C}$ annealing for $30 \mathrm{~s}, 72^{\circ} \mathrm{C}$ extension for $2 \mathrm{~min}$; and then a final $72{ }^{\circ} \mathrm{C}$ extension for $5 \mathrm{~min}$. PCR reactions underwent a $0.9 \mathrm{X}$ SPRIselect single sided size selection purification and eluted with $20 \mu \mathrm{L}$ of nuclease-free water. Biotinylated PCR products were then captured using M-280 Streptavidin Dynabeads ${ }^{\mathrm{tw}}$ and washed $3 \times$ with $10 \mathrm{mM}$ Tris- $\mathrm{HCl}(\mathrm{pH} 7.5) 1 \mathrm{mM}$ EDTA $2 \mathrm{M} \mathrm{NaCl}$ wash buffer prior to final resuspension in $60 \mu \mathrm{L}$ nuclease free water. A second nested PCR was then carried out using $5 \mu \mathrm{L}$ of the streptavidin bead resuspension using $2 \times$ KAPA HiFi HotStart ReadyMix and $0.4 \mu \mathrm{M}$ Nested TCF7 Exon 4 forward primer and 0.4 $\mu \mathrm{M}$ Read 1 reverse primer using 1 cycle of initial denaturation $95^{\circ} \mathrm{C}$ for $5 \mathrm{~min}$, followed by 30 cycles of $98^{\circ} \mathrm{C}$ denaturation for $20 \mathrm{~s}, 63^{\circ} \mathrm{C}$ annealing for $30 \mathrm{~s}, 72^{\circ} \mathrm{C}$ extension for $2 \mathrm{~min}$; and then a final $72{ }^{\circ} \mathrm{C}$ extension for $5 \mathrm{~min}$. The resulting nested PCR underwent a $0.9 \times$ SPRIselect single sided size selection purification and eluted with $20 \mu \mathrm{L}$ of nuclease free water. Amplification was confirmed by TapeStation (Agilent) analysis and Sanger Sequencing (Supplementary Fig. 3) prior to nanopore sequencing.

Nanopore sequencing and analysis. Amplified cDNA libraries were prepared for long-read sequencing using Oxford Nanopore Technologies (ONT) 1D adapter ligation sequencing kit (SQK-LSK109). Three samples were barcoded, pooled and sequenced on a single R9.4.1 PromethION flow cell (FLO-PRO002). MinKNOW live base-calling was performed using Guppy (v4.0.11). The resulting base-called FASTQ files were de-multiplexed by $10 \times$ cell barcodes, using a direct sequence matching strategy described previously ${ }^{73}$. Briefly, forward and reverse-complemented cellbarcode sequences $(16 \mathrm{nt})$ were used to demultiplex the nanopore sequencing reads by scanning the first and last $200 \mathrm{nt}$ of any read longer than $250 \mathrm{nt}$ for a matching sequence, with $<2$ mismatches. Demultiplexed reads were then grouped into separate FASTQ files that were separately assembled de novo using $\mathrm{Canu}^{74}$ (version 1.8; parameters: -Overlapper $=$ minimap - minReadLength $=500$-minOverlapLength $=$ 100 -genomeSize $=1 \mathrm{k}$-nanopore-raw - stopOnReadQuality $=$ false) Demuxed FASTQ reads were then aligned to their respective Canu contigs using Minimap2 $2^{51}$ (version 2.17-r943-dirty) before 'polishing' the consensus sequence using Racon ${ }^{75}$ (version 1.3.3; parameters: - $\mathrm{u}-\mathrm{w} 200-\mathrm{m} 8-\mathrm{x}-1-\mathrm{g}-4$ ). The Minimap2/Racon step was repeated a total of four times. The number of total nanopore reads and demultiplexed reads for each sample is shown in Supplementary Data 5. Reads spanning TCF7 exon \#4, the fusion breakpoint and SPI exon \#3 were classified as 'fusion reads', while reads spanning TCF7 exon \#4 and spanning TCF7 exon \#7 were classified as "canonical reads". Canonical/fusion read counts are summarized in Supplementary Data 5.

Statistical analysis. Statistical analyses were performed using the Prism software (Graphpad v9.0.2 (134)). Kaplan-Meier curves were used for the survival of mouse BMT's with two-sided p values determined by log-rank (Mantel-Cox) test. For the comparison of 2 groups, an unpaired $t$ test with Welch's correction or Mann-Whitney test was used. Data in the luciferase experiments are expressed as means \pm standard deviation (SD). Groups were compared using the post hoc Dunnett T3 multiple comparisons test after Brown-Forsythe ANOVA, $p$ values are adjusted $p$ values. Expression analysis for different subsets of patients was done with a Tukey's multiple comparisons test. For the analysis of the dilution assay we used the ELDA (extreme limiting dilution analysis) software ${ }^{76}$

Reporting summary. Further information on research design is available in the Nature Research Reporting Summary linked to this article.

\section{Data availability}

The data of bulk and single-cell RNA sequencing of 4 T-ALL patients (X09, XB37, XB41, and XB47), nanopore sequencing of X09, together with the single-cell RNA sequencing data of a PDX model of the X09 patient and sequencing data of two patients from the StJude cohort (SJTALL030263 and SJTALL031201), have been deposited within the European Genome-phenome Archive (EGA), which is hosted by the EBI and the CRG. Accession number EGAS00001005097, https://ega-archive.org/datasets/

EGAD00001007010. The Seki et al. RNA-seq data are available at the National Bioscience Database Center, Japan Science and Technology Agency (ID: JGAD00000000090). The whole-genome sequencing data of the four T-ALL samples (X09, XB37, XB41, and XB47) from De Bie et al. ${ }^{40}$ can be accessed through the following link: https://ega-archive.org/ datasets/EGAD00001003951. For restricted data, access can be requested by sending an email to Sofie Demeyer (sofie.demeyer@kuleuven.be) with requests for data use outside academic research, requiring a data access agreement. Processed single-cell data have been deposited with the Zenodo Biorepository (DOI:10.5281/zenodo.4756105, https:// zenodo.org/record/4756105\#.YMbypC0RqqB). For Gene Set Enrichment Analysis, Molecular Signatures Database was used (http://www.gsea-msigdb.org/gsea/msigdb/ index.jsp). Source data are provided with this paper.

\section{Code availability}

Custom R-scripts used have been deposited with the Zenodo Biorepository (DOI:10.5281/zenodo.4756105).

Received: 8 April 2020; Accepted: 18 June 2021; Published online: 06 July 2021

\section{References}

1. De Keersmaecker, K. et al. Exome sequencing identifies mutation in CNOT3 and ribosomal genes RPL5 and RPL10 in T-cell acute lymphoblastic leukemia. Nat. Genet. 45, 186-190 (2013).

2. Li, Y. et al. IL-7 Receptor mutations and steroid resistance in pediatric T cell acute lymphoblastic leukemia: a genome sequencing study. PLoS Med. 13, e1002200 (2016).

3. Degryse, S. et al. JAK3 mutants transform hematopoietic cells through JAK activation causing T-cell acute lymphoblastic leukemia in a bone marrow transplant mouse model. Blood 124, 3092-3100 (2014).

4. Atak, Z. K. et al. Comprehensive analysis of transcriptome variation uncovers known and novel driver events in T-cell acute lymphoblastic leukemia. PLoS Genet. 9, e1003997(2013).

5. Liu, Y. et al. The genomic landscape of pediatric and young adult T-lineage acute lymphoblastic leukemia. Nat. Genet. 49, 1211-1218 (2017).

6. de Bock, C. E. et al. HOXA9 cooperates with activated JAK/STAT signaling to drive leukemia development. Cancer Discov. 8, 616-631 (2018).

7. Vanden Bempt, M. et al. Cooperative enhancer activation by TLX1 and STAT5 drives development of NUP214-ABL1/TLX1-positive T cell acute lymphoblastic leukemia. Cancer Cell 34, 271-285 (2018).

8. Broux, M. et al. Suz12 inactivation cooperates with JAK3 mutant signaling in the development of T-cell acute lymphoblastic leukemia. Blood 134, 1323-1336 (2019).

9. Seki, M. et al. Recurrent SPI1 (PU.1) fusions in high-risk pediatric T cell acute lymphoblastic leukemia. Nat. Genet. 49, 1274 (2017).

10. Van de Wetering, M. et al. The human T cell transcription factor-1 gene. Structure, localization, and promoter characterization. J. Biol. Chem. 267, 8530-8536 (1992).

11. Johnson, J. L. et al. Lineage-determining transcription factor TCF-1 initiates the epigenetic identity of T cells. Immunity 48, 243-257 (2018).

12. Moreau-Gachelin, F., Tavitian, A. \& Tambourin, P. Spi-1 is a putative oncogene in virally induced murine erythroleukaemias. Nature 331, 277-280(1988).

13. Galson, D. L. et al. Mouse beta-globin DNA-binding protein B1 is identical to a proto-oncogene, the transcription factor Spi-1/PU.1, and is restricted in expression to hematopoietic cells and the testis. Mol. Cell. Biol. 13, 2929-2941 (1993).

14. Rosenbauer, F. et al. Acute myeloid leukemia induced by graded reduction of a lineage-specific transcription factor, PU.1. Nat. Genet. 36, 624-630 (2004).

15. Steidl, U. et al. Essential role of Jun family transcription factors in PU.1 knockdown-induced leukemic stem cells. Nat. Genet. 38, 1269-1277 (2006).

16. Zhu, X. et al. The significance of low PU.1 expression in patients with acute promyelocytic leukemia. J. Hematol. Oncol. 5, 22 (2012).

17. Mueller, B. U. et al. Heterozygous PU.1 mutations are associated with acute myeloid leukemia. Blood 100, 998-1007 (2002).

18. Metcalf, D. et al. Inactivation of PU.1 in adult mice leads to the development of myeloid leukemia. Proc. Natl Acad. Sci. USA 103, 1486-1491 (2006).

19. Anderson, M. K., Hernandez-Hoyos, G., Diamond, R. A. \& Rothenberg, E. V. Precise developmental regulation of Ets family transcription factors during specification and commitment to the T cell lineage. Development 126, 3131-3148 (1999)

20. Anderson, M. K., Weiss, A. H., Hernandez-Hoyos, G., Dionne, C. J. \& Rothenberg, E. V. Constitutive expression of PU.1 in fetal hematopoietic progenitors blocks $\mathrm{T}$ cell development at the pro-T cell stage. Immunity 16 , 285-296 (2002).

21. Gupta, P., Gurudutta, G. U., Saluja, D. \& Tripathi, R. P. PU.1 and partners: regulation of haematopoietic stem cell fate in normal and malignant haematopoiesis. J. Cell. Mol. Med. 13, 4349-4363 (2009).

22. Verbeek, S. et al. An HMG-box-containing T-cell factor required for thymocyte differentiation. Nature 374, 70-74 (1995).

23. Weber, B. N. et al. A critical role for TCF-1 in T-lineage specification and differentiation. Nature 476, 63-68 (2011).

24. Del Real, M. M. \& Rothenberg, E. V. Architecture of a lymphomyeloid developmental switch controlled by PU.1, notch and Gata3. Development 140, 1207-1219 (2013).

25. Zhang, J. et al. The genetic basis of early T-cell precursor acute lymphoblastic leukaemia. Nature 481, 157-163 (2012)

26. Park, J. E. et al. A cell atlas of human thymic development defines T cell repertoire formation. Science 367, eaay3224 (2020). 
27. Nusse, R. \& Clevers, H. Wnt/ $\beta$-catenin signaling, disease, and emerging therapeutic modalities. Cell 169, 985-999 (2017).

28. Rosenbauer, F. et al. Lymphoid cell growth and transformation are suppressed by a key regulatory element of the gene encoding PU.1. Nat. Genet. 38, 27-37 (2006).

29. Guo, W. et al. Multi-genetic events collaboratively contribute to Pten-null leukaemia stem-cell formation. Nature 453, 529-533 (2008).

30. Zhu, H. et al. T-ALL leukemia stem cell'stemness' is epigenetically controlled by the master regulator SPI1. Elife 7, e38314 (2018).

31. Rubinfeld B. et al. Stabilization of beta-catenin by genetic defects in melanoma cell lines. Science 275, 1790-1792 (1997).

32. Roos-Weil, D. et al. A recurrent activating missense mutation in Waldenström macroglobulinemia affects the DNA binding of the ETS transcription factor SPI1 and enhances proliferation. Cancer Discov. 9, 796-811 (2019).

33. Fellmann, C. et al. An optimized microRNA backbone for effective singlecopy RNAi. Cell Rep. 5, 1704-1713 (2013).

34. Herrmann, C., Van De Sande, B., Potier, D. \& Aerts, S. i-cisTarget: an integrative genomics method for the prediction of regulatory features and cisregulatory modules. Nucleic Acids Res. 40, e114.(2012).

35. Krishnamurthy, N. \& Kurzrock, R. Targeting the Wnt/beta-catenin pathway in cancer: Update on effectors and inhibitors. Cancer Treat. Rev. 62, 50-60 (2018).

36. Perentesis, J. P. et al. RAS oncogene mutations and outcome of therapy for childhood acute lymphoblastic leukemia. Leukemia 18, 685-692 (2004).

37. Wang, J. et al. Endogenous oncogenic Nras mutation initiates hematopoietic malignancies in a dose- and cell type-dependent manner. Blood 118, 368-379 (2011).

38. Staffas, A., Karlsson, C., Persson, M., Palmqvist, L. \& Bergo, M. O. Wild-type KRAS inhibits oncogenic KRAS-induced T-ALL in mice. Leukemia 29, 1032-1040 (2015).

39. Gocho, Y. et al. Network-based systems pharmacology reveals heterogeneity in LCK and BCL2 signaling and therapeutic sensitivity of T-cell acute lymphoblastic leukemia. Nat. Cancer 2, 284-299 (2021).

40. De Bie, J. et al. Single-cell sequencing reveals the origin and the order of mutation acquisition in T-cell acute lymphoblastic leukemia. Leukemia 32, 1358-1369 (2018).

41. Huang, Y. et al. The leukemogenic TCF3-HLF complex rewires enhancers driving cellular identity and self-renewal conferring EP300 vulnerability. Cancer Cell 36, 630-644.e9 (2019).

42. Yui, M. A. \& Rothenberg, E. V. Developmental gene networks: a triathlon on the course to T cell identity. Nat. Rev. Immunol. 14, 529-545 (2014).

43. Girardi, T., Vicente, C., Cools, J. \& De Keersmaecker, K. The genetics and molecular biology of T-ALL. Blood 129, 2-4 (2018).

44. Mansour, M. R. et al. An oncogenic super-enhancer formed through somatic mutation of a noncoding intergenic element. Science 346, 1373-1377 (2014).

45. de Thé, H., Pandolfi, P. P. \& Chen, Z. Acute promyelocytic leukemia: a paradigm for oncoprotein-targeted cure. Cancer Cell 32, 552-560 (2017).

46. Lewis, A. C. \& Kats, L. M. Non-genetic heterogeneity, altered cell fate and differentiation therapy. EMBO Mol. Med. 13, e12670 (2021).

47. Downward, J. Targeting RAS signalling pathways in cancer therapy. Nat. Rev. Cancer 3, 11-22 (2003).

48. Potter, N. E. et al. Single-Cell mutational profiling and clonal phylogeny in cancer. Genome Res. 23, 2115-2125 (2013).

49. $\mathrm{Xu}, \mathrm{X}$. et al. Single-cell exome sequencing reveals single-nucleotide mutation characteristics of a kidney tumor. Cell 148, 886-895 (2012).

50. Terstappen, L. W. M. M., Huang, S., Safford, M., Lansdorp, P. M. \& Loken, M. R. Sequential generations of hematopoietic colonies derived from single nonlineagecommitted CD34+CD38- progenitor cells. Blood 77, 1218-1227 (1991).

51. Li, H. Minimap2: Pairwise alignment for nucleotide sequences. Bioinformatics 34, 3094-3100 (2018).

52. Gehre, N., et al. A stromal cell free culture system generates mouse pro-T cells that can reconstitute T-cell compartments in vivo. Eur. J. Immunol. 45, 932-942 (2015).

53. Bornschein, S. et al. Defining the molecular basis of oncogenic cooperation between TAL1 expression and Pten deletion in T-ALL using a novel pro-T-cell model system. Leukemia 32, 941-951 (2018).

54. Thorne, N., Inglese, J. \& Auld, D. S. Illuminating Insights into firefly luciferase and other bioluminescent reporters used in chemical biology. Chem. Biol. 17, 646-657 (2010).

55. Dobin, A. et al. STAR: Ultrafast universal RNA-seq aligner. Bioinformatics 29, 15-21 (2013)

56. Anders, S., Pyl, P. T. \& Huber, W. HTSeq-A Python framework to work with high-throughput sequencing data. Bioinformatics 31, 166-169.(2015).

57. Uhrig, S. et al. Accurate and efficient detection of gene fusions from RNA sequencing data. Genome Res. 31, 448-460. (2021).

58. Love, M. I., Huber, W. \& Anders, S. Moderated estimation of fold change and dispersion for RNA-seq data with DESeq2. Genome Biol. 15, 550 (2014).

59. Sergushichev, A. A. An algorithm for fast preranked gene set enrichment analysis using cumulative statistic calculation. bioRxiv. Preprint at https://doi. org/10.1101/060012 (2016).
60. McLaren, W. et al. The ensembl variant effect predictor. Genome Biol. 17, 122 (2016).

61. Zheng, G. X. Y. et al. Massively parallel digital transcriptional profiling of single cells. Nat. Commun. 8, 14049 (2017).

62. Butler, A., Hoffman, P., Smibert, P., Papalexi, E. \& Satija, R. Integrating singlecell transcriptomic data across different conditions, technologies, and species. Nat. Biotechnol. 36, 411-420 (2018).

63. Stuart, T. et al. Comprehensive integration of single-cell data. Cell 177, 1888-1902.e21 (2019)

64. Lun, A. et al. EmptyDrops: distinguishing cells from empty droplets in droplet-based single-cell RNA sequencing data. Genome Biol. 22, 63 (2019).

65. Ilicic, T. et al. Classification of low quality cells from single-cell RNA-seq data Genome Biol. 17, 29 (2016)

66. Hafemeister, C. \& Satija, R. Normalization and variance stabilization of singlecell RNA-seq data using regularized negative binomial regression. Genome Biol. 20, 296 (2019).

67. Korsunsky, I. et al. Fast, sensitive and accurate integration of single-cell data with Harmony. Nat. Methods 16, 1289-1296 (2019).

68. Zappia, L. \& Oshlack, A. Clustering trees: a visualization for evaluating clusterings at multiple resolutions. Gigascience 7, giy083 (2018).

69. McInnes, L., Healy, J., Saul, N. \& Großberger, L. UMAP: Uniform manifold approximation and projection. J. Open Source Softw. 3, 861 (2018).

70. Becht, E. et al. Dimensionality reduction for visualizing single-cell data using UMAP. Nat. Biotechnol. 37, 38-44 (2019).

71. Gu, Z., Eils, R. \& Schlesner, M. Complex heatmaps reveal patterns and correlations in multidimensional genomic data. Bioinformatics 32, 2847-2849 (2016).

72. Korotkevich, G., Sukhov, V. \& Sergushichev, A. Fast gene set enrichment analysis. biorxiv. Preprint at https://doi.org/10.1101/060012 (2016)

73. Singh, M. et al. High-throughput targeted long-read single cell sequencing reveals the clonal and transcriptional landscape of lymphocytes. Nat. Commun. 10, 3120 (2019).

74. Koren, S. et al. Canu: scalable and accurate long-read assembly via adaptive $\kappa-$ mer weighting and repeat separation. Genome Res. 27, 722-736 (2017).

75. Vaser, R., Sović, I., Nagarajan, N. \& Šikić, M. Fast and accurate de novo genome assembly from long uncorrected reads. Genome Res. 27, 737-746 (2017).

76. Hu, Y. \& Smyth, G. K. ELDA: Extreme limiting dilution analysis for comparing depleted and enriched populations in stem cell and other assays. J. Immunol. Methods 347, 70-78 (2009).

\section{Acknowledgements}

This study was supported by a fellowship from the FWO-Vlaanderen (J.D.), Cancer Institute New South Wales (D.R.C.), Children's Cancer Institute (C.D.B.), Steven Walter Children's Cancer Foundation (A.B.), and by grants from the European Research Council (J.C.), FWO-Vlaanderen (J.C.) and the Foundation against Cancer (J.C.). We would like to thank Prof. Dr. Johannes Zuber, IMP-Research Institute of Molecular Pathology $\mathrm{GmbH}$, Austria for providing the LT3GECIR lentiviral miR-E shRNA vector. We would like to thank the St. Jude Biorepository for providing the two SJTALL03263_D1 and SJTALL031201_D1 TCF7-SPI1 fusion case samples. We thank Dr. Emma Johansson Beves from the Flow Cytometry Facility, Mark Wainwright Analytical Centre at UNSW, Sydney for assistance with flow cytometry and sorting experiments.

\section{Author contributions}

J.D., Q.V.T., and C.D.B. performed research, analyzed data, and wrote the manuscript. S.D., D.R.C., T.G.A., I.W.D., and J.S. performed research, nanopore sequencing, and bioinformatics data analysis. W.T., B.S., E.G., S.P., S.O., A.B., V.Z., H.M., R.B.L., E.P.O., M.M.G., R.A. D., B.C., G.M.M., and O.G. performed research and analyzed data. H.S., N.B., K.I., I.K. J.T. and A.U. collected samples and analyzed data. D.C. supervised the analysis of the single-cell data. R.B.L. and R.A.D. supervised and assisted analysis of in vivo inducible knockdown mouse work. J.C. and C.D.B. supervised the study, analyzed data, and wrote the manuscript.

\section{Competing interests}

The authors declare no competing interests.

\section{Additional information}

Supplementary information The online version contains supplementary material available at https://doi.org/10.1038/s41467-021-24442-9.

Correspondence and requests for materials should be addressed to J.C. or C.E.d.B.

Peer review information Nature Communications thanks David Kent, Charles Mullighan and Bo Porse for their contribution to the peer review of this work

Reprints and permission information is available at http://www.nature.com/reprints

Publisher's note Springer Nature remains neutral with regard to jurisdictional claims in published maps and institutional affiliations. 
(c) (i) Open Access This article is licensed under a Creative Commons Attribution 4.0 International License, which permits use, sharing, adaptation, distribution and reproduction in any medium or format, as long as you give appropriate credit to the original author(s) and the source, provide a link to the Creative Commons license, and indicate if changes were made. The images or other third party material in this article are included in the article's Creative Commons license, unless indicated otherwise in a credit line to the material. If material is not included in the article's Creative Commons license and your intended use is not permitted by statutory regulation or exceeds the permitted use, you will need to obtain permission directly from the copyright holder. To view a copy of this license, visit http://creativecommons.org/ licenses/by/4.0/.

(C) The Author(s) 2021 